https://helda.helsinki.fi

Twisted geometries, twistors, and conformal transformations

\title{
Långvik, Miklos
}

2016-07-26

Långvik , M \& Speziale , S 2016 , ' Twisted geometries, twistors, and conformal transformations ' , Physical Review D , vol. 94 , no. 2 , 024050 . https://doi.org/10.1103/PhysRevD.94.024050

http://hdl.handle.net/10138/184152

https://doi.org/10.1103/PhysRevD.94.024050

cc_by

publishedVersion

Downloaded from Helda, University of Helsinki institutional repository.

This is an electronic reprint of the original article.

This reprint may differ from the original in pagination and typographic detail.

Please cite the original version. 
PHYSICAL REVIEW D 94, 024050 (2016)

\title{
Twisted geometries, twistors, and conformal transformations
}

\author{
Miklos Långvik $^{1,2}$ and Simone Speziale ${ }^{1}$ \\ ${ }^{1}$ Centre de Physique Théorique, CNRS UMR 7332, AMU \& USTV, \\ Luminy Case 907, 13288 Marseille, France \\ ${ }^{2}$ Department of Physics, University of Helsinki, P.O. Box 64, FIN-00014 Helsinki, Finland
}

(Received 16 February 2016; published 26 July 2016)

\begin{abstract}
The twisted geometries of spin network states are described by simple twistors, isomorphic to null twistors with a timelike direction singled out. The isomorphism depends on the Immirzi parameter $\gamma$ and reduces to the identity for $\gamma=\infty$. Using this twistorial representation, we study the action of the conformal group $\mathrm{SU}(2,2)$ on the classical phase space of loop quantum gravity, described by twisted geometry. The generators of translations and conformal boosts do not preserve the geometric structure, whereas the dilatation generator does. It corresponds to a one-parameter family of embeddings of $\mathrm{T}^{*} \mathrm{SL}(2, \mathrm{C})$ in twistor space, and its action preserves the intrinsic geometry while changing the extrinsic one-that is the boosts among polyhedra. We discuss the implication of this action from a dynamical point of view and compare it with a discretization of the dilatation generator of the continuum phase space, given by the Lie derivative of the group character. At leading order in the continuum limit, the latter reproduces the same transformation of the extrinsic geometry, while also rescaling the areas and volumes and preserving the angles associated with the intrinsic geometry. Away from the continuum limit, its action has an interesting nonlinear structure but is in general incompatible with the closure constraint needed for the geometric interpretation. As a side result, we compute the precise relation between the extrinsic geometry used in twisted geometries and the one defined in the gauge-invariant parametrization by Dittrich and Ryan and show that the secondary simplicity constraints they posited coincide with those dynamically derived in the toy model of [Classical Quantum Gravity 32, 195015 (2015)].
\end{abstract}

DOI: 10.1103/PhysRevD.94.024050

\section{INTRODUCTION}

While loop quantum gravity (for a recent monograph, see Ref. [1]) is a background-independent approach to quantum gravity, the internal Minkowski metric plays a key role in identifying the local gauge group of the theory, $\mathrm{SL}(2, \mathbb{C})$ in the covariant formulation and $\mathrm{SU}(2)$ through the use of Ashtekar-Barbero variables. The Minkowski metric has also a group $\mathrm{SU}(2,2)$ of (a four-fold cover of) conformal isometries. In this paper, we study the action of this group on the phase space of loop quantum gravity on a fixed graph. This space, that corresponds to the kinematical semiclassical description of the theory, has been shown to be described by a collection of polyhedra [2-5], which defines a discrete metric structure with intrinsic and extrinsic components, called twisted geometry. These can in turn be parametrized in terms of twistors, and it is the Hamiltonian action of $\mathrm{SU}(2,2)$ on twistor space that we use for our study.

The interest in studying conformal transformations on this space is two-fold. On the one hand, conformal transformations play an important role in classical general relativity, and it would be very useful to have any of their applications available in the study of loop quantum gravity, such as identifying the splitting between the causal structure and conformal factor of the metric or the behavior of transition amplitudes under dilatations. As twisted geometries are discrete, we may expect difficulties in realizing conformal transformations. Indeed, we know from results in Regge calculus that one cannot realize in a discrete setting conformal transformations in the usual sense of rescaling distances while preserving angles [6], ultimately for the simple reason that angles are determined themselves by edge lengths and/or areas. As we show in this paper, using twisted geometries and the twistorial representation of the conformal group allows one to go partially beyond this result; while the generators of translations and conformal boosts are not compatible with the constraints present in twisted geometries, the generator of dilatations is, and can thus be meaningfully realized on the phase space. Its geometric interpretation is, however, counterintuitive: first, the 3D intrinsic geometry is invariant, including areas and volumes; second, only the extrinsic geometry changes, the orbits giving linear shifts of the boosts among the polyhedra. This unconventional behavior for a dilatation may appear puzzling, but it is a natural consequence of the relation between the variables of loop quantum gravity and the Lorentz algebra. In fact, the intrinsic geometry of loop quantum gravity is built out of angular momentum operators, and these are invariant under the dilatations of their embedding conformal group. It is only the extrinsic geometry, built out of the holonomies, that can be affected by these dilatations. Since changing the extrinsic geometry without changing the 
intrinsic one affects the spacetime curvature, our result suggests that the $\operatorname{SU}(2,2)$ dilatation generator can play a dynamical role in the theory, something we briefly comment upon. It is then natural to ask whether a more conventional dilatation generator exists on the phase space of twisted geometries. The situation can be compared with a more geometric notion of dilatations, rescaling distances while preserving angles, such as symplectic dilatations in the phase space of connections and triads of the continuum theory. While dilatations in this sense do not exist on the phase space of a fixed graph because it has compact directions, nor can they be expected from the Regge calculus analysis, it is possible to define a discrete version of the continuum symplectic dilatations. The nonlinearity of its action can be organized in an interesting way, and its effect on the geometry can be studied explicitly. At leading order in the continuum limit, the latter reproduces the same transformation of the extrinsic geometry, while also rescaling the areas and volumes and preserving the angles associated with the intrinsic geometry. However, away from the continuum limit, its action is highly nonlinear and, in particular, is in general incompatible with the closure constraint needed for the geometric interpretation.

The second line of interest in our question is more formal. In their initial formulation by Penrose, spin networks were conceived to describe only the conformally invariant part of a quantum spacetime, via the angles. To introduce distances, he envisaged an extension to the Poincare group or, since the latter is not semisimple, to the conformal group of Minkowski spacetime. This program famously led to twistor theory [7], based on the double cover of the conformal symmetry group of Minkowski spacetime $\mathrm{SO}(4,2)$, or its double cover SU $(2,2)$. Later on, $\mathrm{SU}(2)$ spin networks found a key role in loop quantum gravity, where they provide an orthonormal basis of the theory. In their use in quantum gravity, the norm of the angular momentum is interpreted as an area eigenvalue, thus introducing a notion of scale and distances per se. Extensions to $\mathrm{SL}(2, \mathbb{C})$ are also commonly used in linking loop quantum gravity to the spin foam formalism for transition amplitudes. It is nonetheless still an open and intriguing question to develop Penrose's original program and establish a precise relation between $\mathrm{SU}(2,2)$ spin networks and the SU(2) ones used in LQG. Such SU $(2,2)$ spin networks would be based on twistors in a similar way as the SU(2) ones are based on spinors, and to understand their relation precisely, it is useful to first clarify how the conformal symmetry of twistor space is broken in the way loop quantum gravity uses this space. We answer here this question at the classical level, showing that it is the area-matching constraint, responsible for reducing the twistor phase space to the $\operatorname{SL}(2, \mathbb{C})$ phase space, that partially breaks the conformal symmetry, with only the action of dilatations remaining. Our results will be then useful in future works concerned with understanding the relation at the quantum level.

The paper is organized as follows. In Sec. II, we provide a short overview of the link between twistor theory and loop quantum gravity. This allows us to introduce the representation of the conformal group that we will use later on but also to provide an overview of the state of the art of twisted geometries, that has seen many remarkable developments in the last couple of years. In Sec. III, we show what the linear, primary simplicity constraints used in spin foam models for loop quantum gravity [8] mean from the point of view of twistor theory. The Lorentz-invariant part ("diagonal simplicity") selects twistors with helicity linked to the Lorentz Casimirs, a constraint breaking full conformal invariance. While the helicity is nonvanishing, the constraint structure makes these twistors isomorphic to null twistors. The isomorphism depends on the Immirzi parameter $\gamma$ (reducing to the identity for $\gamma=\infty$ ), and we refer to these as $\gamma$-null twistors. The non-Lorentz-invariant part ("off-diagonal simplicity"), depending on a gauge-fixing time direction, selects a special point along the $\gamma$-null twistor's null ray intersecting the time direction. The resulting simple twistors (in the sense that they satisfy the simplicity constraints of loop quantum gravity) identify a unique spacelike plane, that in the geometric reconstruction is used to define the faces of the polyhedra of twisted geometries. In Sec. IV, we compute the action of the conformal group on twisted geometries. Of the non$\mathrm{SL}(2, \mathbb{C})$ generators of $\mathrm{SU}(2,2)$, only the dilatation is compatible with the various geometric constraints (area matching, simplicity, and closure). Its orbits preserve the fluxes and change only the holonomy. At the SU(2) level, the change is a linear shift of the twist angle; this transformation thus leaves unaffected the whole 3D geometry and only changes the embedding of the SU(2) holonomy in the covariant phase space by a linear shift. At the $\operatorname{SL}(2, \mathbb{C})$ level, again the transformation only affects the covariant version of the twist angle, which now carries the interpretation of boosts among adjacent polyhedra, that is of extrinsic curvature. Defining the extrinsic curvature in this way requires fixing the time gauge, and it is desirable to have a completely gauge-independent test of this action. To that end, we consider the alternative description of extrinsic geometry by Dittrich and Ryan [9], here extended to the Lorentzian signature. The latter is fully gauge invariant but depends on a choice of edge per face of the polyhedron and gives a unique boost only for shape-matched configurations. We show that also the extrinsic curvature defined in this way transforms in the same way under a $\mathrm{SU}(2,2)$ dilatation. As a side result, we work out the exact relation between the two definitions of boost dihedral angles, and we prove that they coincide when certain secondary simplicity constraints are satisfied. Such secondary constraints were derived dynamically using a toy model in Ref. [10], and our analysis here shows also that they 
precisely coincide with (the Lorentzian extension of) those defined in Ref. [9].

Finally, in Sec. V, we compare these transformations with those induced by a direct discretization of the continuum connection-triad symplectic dilatations, obtaining the results anticipated above. Our discretization takes the form of a Lie derivative of the group character, and when interpreted in terms of spinors, it acts as a boost in mixing the source and target spinors. In spite of its simplicity, its action on holonomies and fluxes is completely nonlinear and has no resemblance with usual dilatations. This is unavoidable, as the discrete phase space has compact directions, so there is no usual meaning of linear dilatations. On the other hand, the generator has the property of preserving the symplectic structure, and all transformed quantities recover the expected behavior in the continuum limit.

All our results, computing the action of $\mathrm{SU}(2,2)$ dilatations, the relation between the different boost dihedral angles in the literature and proving the equivalence of different secondary simplicity constraints, as well as computing the finite action of the discrete holonomy-flux dilatation, rely heavily on the use of twistorial formalism and are a demonstration of its usefulness to loop quantum gravity.

We use a mostly plus convention for the Minkowski metric, and indices $I=0,1,2,3$. A, $B=0,1$ are spinorial indices, and $\alpha=1,2,3,4$ are twistorial indices.

\section{TWISTORS AND TWISTED GEOMETRIES}

A twistor can be described as a pair of spinors, $Z^{\alpha}=\left(\omega^{A}, i \bar{\pi}_{\dot{A}}\right) \in \mathbb{C}^{2} \oplus \overline{\mathbb{C}}^{2 *}=: \mathbb{T}$. It has a dual, $\bar{Z}_{\alpha}=$ $\left(-i \pi_{A}, \bar{\omega}^{\dot{A}}\right)$, which defines a pseudo-Hermitian norm of signature $(++--)$,

$$
Z^{\alpha} \bar{Z}_{\alpha}=2 \operatorname{Im}\left(\pi_{A} \omega^{A}\right),
$$

preserved by $\mathrm{SU}(2,2)$ transformations. It is well known [7] that these transformations can be realized by Hamiltonian vector fields, if we equip the space with canonical Poisson brackets,

$$
\left\{Z^{\alpha}, \bar{Z}_{\beta}\right\}=i \delta_{\beta}^{\alpha} \text {. }
$$

In Penrose's abstract index convention (see Appendix B), the generators of $\mathrm{SU}(2,2)$ can be written as

$$
\begin{aligned}
J^{I J} & =\omega^{(A} \pi^{B)} \epsilon^{\dot{A} \dot{B}}+\mathrm{cc}, \quad P^{I}=i \pi^{A} \bar{\pi}^{\dot{A}}, \\
C^{I} & =i \omega^{A} \bar{\omega}^{\dot{A}}, \quad D=\operatorname{Re}\left(\pi_{A} \omega^{A}\right),
\end{aligned}
$$

and their finite action on a phase space function $f=f\left(\omega^{A}, \pi^{A}\right)$ is realized via the exponential map,

$$
\begin{aligned}
& \exp \left(\epsilon_{I J} M^{I J}\right) \triangleright f:=\exp \left(\epsilon_{I J}\left\{M^{I J}, \cdot\right\}\right) f \\
& \quad=f+\sum_{n=1}^{\infty} \frac{1}{n !}\left\{\epsilon_{I J} M^{I J}, \ldots,\left\{\epsilon_{K L} M^{K L}, f\right\}\right\},
\end{aligned}
$$

where $\left\{\epsilon_{I J} M^{I J}, \ldots,\left\{\epsilon_{K L} M^{K L}, f\right\}\right\}$ is the $n$-fold nested bracket of the generator $M^{I J}$ with $f$. Half the twistor norm, $s:=\operatorname{Im}\left(\pi_{A} \omega^{A}\right)$, is called helicity of the twistor, ${ }^{1}$ since

$$
W^{I}=-s P^{I},
$$

where $W^{I}:=1 / 2 \epsilon^{I J K L} P_{J} J_{K L}$ is the Pauli-Lubanski vector. The complex scalar $\pi_{A} \omega^{A}=D+i s$, for which we also use the index-free notation $[\pi|\omega\rangle$, is invariant under the Lorentz subalgebra. See Appendix B for more details on the indexfree notation for spinors and their dual.

The representation of the conformal algebra constructed in this way is not the most general one, since the algebra has 15 dimensions, whereas the carrying space $\mathbb{T}$ has only eight dimensions. In fact, the generators (3) are not independent; $P^{I}$ and $C^{I}$ are null and related to $D$ and the Lorentz generators by

$2 P^{[I} C^{J]}=(D \mathbb{1}+s \star) J^{I J}, \quad P^{I} C_{I}=-\left(D^{2}+s^{2}\right)$,

where $\star$ is the Hodge star. Accordingly, the three Casimir operators of $\mathfrak{g} \mathfrak{t}(2,2)$ (see the Appendix for definitions) are not independent; they are all proportional to the only conformally invariant quantity in $\mathbb{T}$, the helicity:

$$
\mathcal{C}^{(2)}=6 s^{2}, \quad \mathcal{C}^{(3)}=-6 s^{3}, \quad \mathcal{C}^{(4)}=6 s^{4} .
$$

Hence, the representation of the algebra on twistor space is special, and furthermore reducible, with irreducibles labelled by $s$. In this sense, Penrose refers to twistors as the spinors of the conformal group.

Irreducible representations with independent real values of the Casimirs can be obtained working with the larger carrying space $\mathbb{T}^{2}$ made of pairs of twistors $(Z, \tilde{Z})$, with generators constructed by linearity. This space has now four conformally invariant quantities, given by the two helicities plus the pseudo-Hermitian product $\bar{Z}_{\alpha} \tilde{Z}^{\alpha}$, and the Casimirs are independent functions of these four variables. $\mathbb{T}^{2}$ has been considered in the twistor literature [11,12], among other things in relation to ambitwistor theory and to build representations of massive particles. But as shown in Ref. [13], $\mathbb{T}^{2}$ has another interesting property that links

\footnotetext{
${ }^{1}$ In the twistor literature, the helicity is usually given by the real part. The difference comes from the extra $i$ used here in the definition of the twistor. In this way, we can match Dirac's conventions for a bispinor and bridge more easily with the notation used in loop quantum gravity. Notice also that we use metric conventions with mostly plus, and thus we map vectors to anti-Hermitian matrices; see Appendix A.
} 
it to loop quantum gravity: it contains $T^{*} \mathrm{SL}(2, \mathbb{C})$ as a symplectic submanifold, obtained by Hamiltonian reduction with respect to the first class constraint

$$
C:=[\pi|\omega\rangle-[\tilde{\pi}|\tilde{\omega}\rangle=0,
$$

referred to as the (complex) area-matching condition. Explicitly, we take

$$
\begin{aligned}
J^{I J} & =\omega^{(A} \pi^{B)} \epsilon^{\dot{A} \dot{B}}+c c, \\
h^{A}{ }_{B} & =\frac{\tilde{\omega}^{A} \pi_{B}-\tilde{\pi}^{A} \omega_{B}}{\sqrt{[\pi|\omega\rangle} \sqrt{[\tilde{\pi}|\tilde{\omega}\rangle}}, \\
\tilde{J}^{I J} & =-\tilde{\omega}^{(A} \tilde{\pi}^{B)} \epsilon^{\dot{A} \dot{B}}+c c,
\end{aligned}
$$

and following the conventions of Ref. [13], we take opposite signs for the twistor brackets ${ }^{2}$ :

$$
\left\{\pi_{A}, \omega^{B}\right\}=\delta_{A}^{B}=-\left\{\tilde{\pi}_{A}, \tilde{\omega}^{B}\right\} .
$$

It is easy to check that (9) are $C$ invariant. When the constraint (8) is satisfied, the generators are related by the adjoint action of $h$ and thus (9) span a 12-dimensional submanifold of $\mathbb{T}^{2}$, for which one recovers the Poisson brackets of $T^{*} \operatorname{SL}(2, \mathbb{C})$ with $J$ and $\tilde{J}$ acting respectively as left-invariant and right-invariant vector fields on the group manifold, parametrized by $h$ as a left-handed group element. This symplectic reduction holds provided $[\pi|\omega\rangle \neq 0$, which we exclude from our analysis from now on and is 2 to 1 ; there is a $\mathbb{Z}^{2}$ symmetry associated with the simultaneous sign flip of the spinors. See Refs. [13-15] for more details.

The symplectic manifold $T^{*} \operatorname{SL}(2, \mathbb{C})$ is the building block of projected spin networks [16], which appear as boundary states of covariant spin foam models $[17,18]$. There, a copy of $T^{*} \operatorname{SL}(2, \mathbb{C})$ decorates each link of an oriented graph, and the orientation is used to associate unambiguously $Z$ to the source node and $\tilde{Z}$ to the target. Each twistor is then subject to the primary simplicity constraints, which reduce the covariant boundary states to those relevant for the $\mathrm{SU}(2)$ spin networks of loop quantum gravity. The constraints are deduced from a discretization of the gravitational action in Ashtekar variables and relate the tetrad to the bivector $B$ which is the field canonically conjugated to the connection. To represent the constraints at the discrete level, we introduce a time direction $N^{I}(n)$ on each node $n$ of the graph. In their linear form introduced in Ref. [19] (see also Refs. [8,20,21]), the primary simplicity constraints read

\footnotetext{
${ }^{2}$ This is convenient for the geometric interpretation of the theory. Switching to the alternative conventions with equal-sign brackets also used in the literature is straightforward, via the map $\omega^{A} \mapsto \pi^{A}, \pi^{A} \mapsto-\omega^{A}$.
}

$$
N_{I} B^{I J}=0,
$$

for all bivectors $B$ associated with links sharing the node $n$, and imply simplicity of $B$, namely $\epsilon_{I J K L} B^{I J} B^{K L}=0$. The bivector $B$ is related to the Lorentz generators via $B=(\mathbb{1}-\gamma \star) J$, where $\gamma \in \mathbb{R}$ is the Immirzi parameter. See Refs. [8,22,23] for details and Ref. [24] for extensions to the case of a null hypersurface.

It is customary the fix the time gauge $N^{I}=(1,0,0,0)$, but the construction extends to an arbitrary gauge [22]. In this gauge, we identify the left-handed generators with 3 -vectors $\Pi^{i}$, the real and imaginary parts of which are rotations $L^{i}$ and boosts $K^{i}$, via $\Pi^{i}=\left(L^{i}+i K^{i}\right) / 2$. The normal $N^{I}$ is conserved by the canonical SU(2) subgroup of the Lorentz group and allows us to define the Hermitian scalar product between spinors, $\langle\omega \mid \pi\rangle:=\delta^{A \dot{A}} \pi_{A} \bar{\omega}_{\dot{A}}$. We will also use the short-hand notation $\|\omega\|^{2}:=\langle\omega \mid \omega\rangle$ for the norm.

The constraints (11) read $K^{i}+\gamma L^{i}=0$ and are equivalent to imposing the matching of left-handed and righthanded sectors up to a phase,

$$
\Pi^{i}=-e^{i \theta} \bar{\Pi}^{i}, \quad \gamma=\cot \frac{\theta}{2} .
$$

Using (3), the constraints have simple spinorial equivalents $[13,14]$,

$$
F_{1}=D-\gamma s=0, \quad F_{2}=\langle\omega \mid \pi\rangle=0 .
$$

The constraint $F_{1}$, real and Lorentz invariant, is solved posing

$$
[\pi|\omega\rangle=(\gamma+i) j, \quad j \in \mathbb{R}
$$

and fixes the relative global phase between the spinors. $F_{2}$, which explicitly depends on the chosen time direction, implies that one spinor is proportional to the parity transform of the other, $\pi_{A} \propto \delta_{A \dot{A}} \bar{\omega}^{\dot{A}}$. Putting the two conditions together, the constraints can be solved expressing one spinor in terms of the other and the norm $j$ :

$$
\pi_{A}=\frac{(\gamma+i) j}{\|\omega\|^{2}} \delta_{A \dot{A}} \bar{\omega}^{\dot{A}} .
$$

There is an alternative parametrization of the solution space, motivated by the canonical structure of the constraints: while $F_{2}$ is second class, $F_{1}$ is first class. A good coordinate among its orbits is the $\mathrm{SU}(2)$ norm $\|\omega\|^{2}$, and the $F_{1}$-gauge-invariant spinor is

$$
z^{A}:=\sqrt{2 j} \frac{\omega^{A}}{\|\omega\|^{i \gamma+1}}, \quad\|z\|=\sqrt{2 j} .
$$

Here, $j \neq 0$ by assumption, and we have restricted to $j>0$ using the $\mathbb{Z}^{2}$ symmetry of the symplectic reduction by $C$. See Ref. [13] for details. 
The solution space is thus a five-dimensional manifold $\mathbb{S}_{\gamma}^{0}$, dependent on the Immirzi parameter (via $\theta$ ) and the choice of the time gauge, that can be parametrized by one of the two Lorentzian spinors, plus the norm $j$, or alternatively, by the reduced spinor $z^{A}$, plus the norm $\|\omega\|$ :

$$
\mathbb{S}_{\gamma}^{0}=\left\{\omega^{A}, j\right\}=\left\{z^{A},\|\omega\|\right\}
$$

The two norms $j$ and $\|\omega\|$ play complementary roles in the geometric interpretation of loop quantum gravity: $j$ is the area of the face dual to the link, while $\|\omega\|$ is related to the extrinsic curvature, as we will recall below. We refer to twistors satisfying the simplicity constraints (13), or equivalently (15), as simple twistors. ${ }^{3}$

On a link, we impose the same set of constraints on both source and target twistors,

$$
\frac{z^{A}}{\|z\|}=\frac{\omega^{A}}{\|\omega\|^{i \gamma+1}}, \quad \frac{\tilde{z}^{A}}{\|\tilde{z}\|}=\frac{\tilde{\omega}^{A}}{\|\tilde{\omega}\|^{i \gamma+1}}
$$

with induced Poisson brackets $\left\{z^{A}, \bar{z}^{\bar{B}}\right\}=i \delta^{A \dot{B}}=$ $-\left\{\tilde{z}^{A}, \overline{\tilde{z}}^{\bar{B}}\right\}$ and reduced area-matching condition $C=\|z\|^{2}-\|\tilde{z}\|^{2}=0$. The latter implies $\|z\|^{2}=\|\tilde{z}\|^{2}=$ $2 j$ and reduces this $\mathbb{C}^{4}$ symplectic space to $T^{*} \mathrm{SU}(2)$, as shown in Ref. [25], parametrized by

$$
\begin{aligned}
\vec{X} & =\frac{1}{2}\langle z|\vec{\sigma}| z\rangle, \\
g & =\frac{|\tilde{z}\rangle\langle z|+| \tilde{z}][z \mid}{\|z\|\|\tilde{z}\|}, \\
\overrightarrow{\tilde{X}} & =-\frac{1}{2}\langle\tilde{z}|\vec{\sigma}| \tilde{z}\rangle,
\end{aligned}
$$

where $\vec{\sigma}$ is the vector of Pauli matrices and $X^{2}=\tilde{X}^{2}=j^{2}$. Again, $X$ and $\tilde{X}$ are related on shell of $C$ by the adjoint action of $g$ and act as right-invariant and left-invariant vector fields.

That is, twistors constrained by the area-matching and primary simplicity constraints describe the phase space $T^{*} \mathrm{SU}(2)$ of loop quantum gravity. When $\mathrm{SU}(2)$ gauge invariance holds, by means of the closure conditions $\sum_{l \in n} X_{l}=0$ at each node of the graph, ${ }^{4}$ the space describes twisted geometries, a generalization of Regge geometries with polyhedra glued along faces that have unique areas but a priori different shapes $[2,5,26]$. The intrinsic geometry is defined from the shapes of the polyhedra, reconstructed from the $X_{l}$ vectors interpreted as normals to the faces [5]. The extrinsic geometry between the polyhedra can be

\footnotetext{
${ }^{3}$ The term "simple twistors" is also used in the twistor literature, but in reference to a bitwistor being simple in the same sense of a simple bivector as defined above.

${ }^{4}$ Here, $\tilde{X}_{l}$ is to be used if $l$ is incoming instead of outgoing from $n$.
}

defined via a Lorentzian dihedral angle, that is the boost relating the four-dimensional normals between adjacent polyhedra [13], ${ }^{5}$

$$
\cosh \Xi:=-\tilde{N}_{I} \Lambda_{J}^{I}(h) N_{J},
$$

where $\Lambda(h)$ is the Lorentz transformation induced by the parallel transport $h$ in the vectorial representation. On shell of the simplicity constraints, this gives

$$
\Xi=\ln \frac{\|\omega\|^{2}}{\|\tilde{\omega}\|^{2}}
$$

Notice that at first sight the extrinsic geometry, described by the boost $\Xi$, is missing in the reduced $\mathrm{SU}(2)$ phase (19). However, this is not the case; the reduced holonomy carries information about boosts. To understand this subtle but crucial point, notice first that the $\mathrm{SU}(2)$ obtained from the reduction is not (the double cover of) the rotation subgroup of $\operatorname{SL}(2, \mathbb{C})$ but is instead embedded in $T^{*} \operatorname{SL}(2, \mathbb{C})$ in a nontrivial way, which in particular spans also the boost directions. To understand this, let us look at the value on shell of the simplicity constraints of the left-handed connection, which is given by

$$
h=\frac{e^{-(1+i \gamma) \frac{\bar{E}}{2}}|\tilde{z}\rangle\left\langle z\left|+e^{(1+i \gamma) \overline{\bar{\Sigma}}}\right| \tilde{z}\right][z \mid}{\|z\|\|\tilde{z}\|} .
$$

This expression shows that the reduced SU(2) is related to the left-handed $S U(2)$ in a $\Xi$-dependent way. Recall that in the continuum, the Ashtekar-Barbero connection is defined as $A^{i}=-\frac{1}{2} \epsilon^{i j k} \omega^{j k}+\gamma \omega^{0 i}$, where $\omega^{I J}$ is the Lorentz connection, and its boost part $\omega^{0 i}$ gives the extrinsic curvature. Here, $i=1,2,3$ is a spatial index in the internal space. In terms of the left-handed part $\omega^{i}:=(\mathbb{1}+i \star) \omega^{0 i}$, this can be rewritten as $\omega^{i}=(1+i \gamma) \omega^{0 i}-i A^{i}$. Equation (22) is precisely a discrete version of this relation and shows that the reduced holonomy $g$ is indeed a discrete version of the Ashtekar-Barbero connection. ${ }^{6}$

The above mixing of rotations and boosts, central to the use of Ashtekar-Barbero variables, is the consequence of the $\gamma$-phase in the simplicity constraints (12), or equivalently in the spinors (16). It shows that the SU(2) discrete data do carry information on the extrinsic geometry, namely the Lorentz boost $\Xi$. In order to extract this information, however, like in the continuum, one needs the embedding of the SU(2) phase space in the covariant one. The latter is

\footnotetext{
${ }^{5}$ Alternatively, it can be defined via the edge vectors as done in Ref. [9]. The precise relation and on-shell equivalence of these two constructions will be proved below in Sec. IV B.

${ }^{6}$ To avoid confusion, let us stress that it is not the holonomy of the Ashtekar-Barbero connection; that wold require imposing the simplicity constraints at every point of the path, as suggested in Ref. [27], but that is a procedure not available if one works, as in here, with a discrete phase space associated with a fixed graph.
} 
provided by the secondary simplicity constraints, which express $\omega^{i j}=\omega^{i j}(E)$ and thus allow us to read the extrinsic curvature from the reduced data, $\omega^{0 i}=\omega^{0 i}(A, E)$. As discussed in Ref. [13] and shown explicitly in Ref. [10], the discrete equivalent of this mechanism goes as follows: the secondary simplicity constraints form a second class pair with $F_{1}$, and thus imposing them gives an explicit gauge fixing of the orbits of $F_{1}$. This gauge fixing provides a function $\Xi_{l}=\Xi_{l}\left(\left\{z_{l}\right\}\right)$ (typically non-local on the graph), that is a way to express the extrinsic geometry in terms of the reduced SU(2) data, as desired.

To explicitly write the secondary constraints, and provide explicit formulas for this procedure, it is best to work in terms of $\mathrm{SU}(2)$ gauge-invariant quantities. A natural and familiar basis for these are the Wilson loops $\operatorname{Tr} \prod_{l \in f} h_{l}$ on the graph. Using spinors, an alternative convenient basis is provided by the set over all nodes of the products $\left\langle z_{i} \mid z_{j}\right\rangle$ and $\left[z_{i}\left|z_{j}\right\rangle\right.$ of spinors in the half-links $i, j$ sharing a node (in the rest of this section, we will not use internal indices $i$ anymore, so we use $i, j$, etc., to refer to half-links). This basis, ${ }^{7}$ introduced in Ref. [4] for loop quantum gravity, can be given a geometric parametrization as follows [29]:

$$
\begin{aligned}
& \left\langle z_{i} \mid z_{j}\right\rangle=\sqrt{4 j_{i} j_{j}} \cos \frac{\phi_{i j}}{2} e^{-\frac{i}{2}\left(\alpha_{j}^{i}-\alpha_{i}^{j}\right)}, \\
& {\left[z_{i}\left|z_{j}\right\rangle=\epsilon_{i j} \sqrt{4 j_{i} j_{j}} \sin \frac{\phi_{i j}}{2} e^{\frac{i}{2}\left(\alpha_{j}^{i}+\alpha_{i}^{j}\right)} .\right.}
\end{aligned}
$$

Here, we assumed that both links $i$ and $j$ are outgoing from the node, ${ }^{8}$ and $\epsilon_{i j}= \pm$ is needed for the phases $\alpha_{j}^{i}$ to be defined in $[0,2 \pi)$. The geometric interpretation of these invariants relies on a map from the graph to its dual cellular decomposition, such that each link of the graph is dual to a face which is shared by two polyhedra dual to the nodes connected by the link. Then, recall through (19) that each spinor defines a vector. Then, $j_{i}$ is the norm of this vector, and $\phi_{i j}$ is the angle between the vectors $\vec{X}_{i}$ and $\vec{X}_{j}$. When the closure constraint at a node is satisfied, the vectors define a unique convex and bounded flat polyhedron, the shape and adjacency matrix of which can be reconstructed using for instance the algorithm given in Ref. [5]. ${ }^{9}$ Then, for adjacent faces $(i j), \phi_{i j}$ are the three-dimensional external dihedral angles, $\vec{X}_{i} \times \vec{X}_{j}$ is the edge vector, and taking scalar products between the edge vectors, one can immediately check that $\alpha_{j k}^{i}:=\alpha_{j}^{i}-\alpha_{k}^{i}$ are the 2D angles between the vectors associated with the edges $(i j)$ and $(i k)$

\footnotetext{
${ }^{7}$ Notice that both bases are redundant, the first by the Mandelstam identities associated with the recoupling theory [28], the second by the Plücker relations $\left[z_{i}\left|z_{j}\right\rangle\left[z_{k}\left|z_{l}\right\rangle=\right.\right.$ $\left[z_{i}\left|z_{l}\right\rangle\left[z_{k}\left|z_{j}\right\rangle+\left[z_{i}\left|z_{k}\right\rangle\left[z_{j}\left|z_{l}\right\rangle\right.\right.\right.\right.$. These latter have the further advantage of generating an algebra, with subalgebra $\mathfrak{t}(N)$ generated by the $\left\langle z_{i} \mid z_{j}\right\rangle$ alone.

${ }^{8}$ If one is incoming, the required parity map is explicitly given by $P|z\rangle=-\mid z]$.

${ }^{9}$ See also Ref. [30] for analytic formulas for the 5-valent case.
}

and thus, when they are adjacent, the 2D dihedral angles of the polygon. ${ }^{10}$

In the usual twisted geometry parametrization, one extracts the direction of the normal vectors via the Hopf section

$$
n=n(\zeta)=\frac{1}{\sqrt{1+|\zeta|^{2}}}\left(\begin{array}{cc}
1 & \zeta \\
-\bar{\zeta} & 1
\end{array}\right): S^{2} \mapsto \mathrm{SU}(2),
$$

where $\zeta:=z^{0} / z^{1}$ and similarly for the tilded spinors with $\tilde{\zeta}:=\tilde{z}^{0} / \tilde{z}^{1}$. Then, Eq. (19) can be rewritten as

$$
\begin{aligned}
\vec{X} & =2 j \operatorname{Tr}\left(n \tau_{3} n^{-1} \vec{\tau}\right), \\
g & =\tilde{n} e^{\xi \tau_{3}} n^{-1}, \\
\overrightarrow{\tilde{X}} & =-2 j \operatorname{Tr}\left(\tilde{n} \tau_{3} \tilde{n}^{-1} \vec{\tau}\right),
\end{aligned}
$$

where $\vec{\tau}=-i / 2 \vec{\sigma}$ and $\xi:=2 \arg \tilde{z}^{1}-2 \arg z^{1} \in[0,4 \pi)$ parametrizes the remaining freedom in $g$ at fixed $X$ and $\tilde{X}$. As the directions and the norms are used to reconstruct the metric properties of the individual polyhedra, $\xi$ is the natural candidate to describe the extrinsic geometry among them, which, as we argued above, is in covariant terms described by the boost $\Xi$. However, there are two nontrivial aspects concerning the exact relation between $\xi$ and $\Xi$ : (i) $\xi$ is not $\mathrm{SU}(2)$ gauge invariant, while $\Xi$ is; (ii) $\xi$ is an $\mathrm{SU}(2)$ angle, while $\Xi$ is a Lorentzian boost. The first issue was initially dealt with simply by assuming to work in a fixed gauge $[2,3]$. An alternative proposed in Ref. [9] is to consider a gaugeinvariant definition of the twist angle via the scalar product between the edge vectors associated to the same edge in the two frames of the source and target nodes:

$$
\cos \xi_{j k}^{i}:=\frac{\left(\vec{X}_{\tilde{l}} \times \vec{X}_{k}\right) \cdot g_{i} \triangleright\left(\vec{X}_{i} \times \vec{X}_{j}\right)}{\left|X_{\tilde{\imath}} \times X_{k}\right|\left|X_{i} \times X_{j}\right|} .
$$

The price to pay for explicit gauge invariance is the edge dependence: only for shape-matched configurations, the above angle is independent of the choice of edge. Thanks to the spinorial gauge-invariant basis (23), it is also possible to compute the SU(2) gauge-invariant twist angle [29] as follows,

$$
\begin{aligned}
\xi_{j k}^{i}:= & \alpha_{k}^{\tilde{i}}-\alpha_{j}^{i}=\xi_{i}+\varphi_{i k}^{+-}-\varphi_{i k}^{--}-\varphi_{i j}^{+-} \\
& +\varphi_{i j}^{--}+\frac{\pi}{2}\left(\epsilon_{i k}-\epsilon_{i j}\right)
\end{aligned}
$$

where $\varphi_{i j}^{A B}$ is the phase of the matrix elements $\left\langle A\left|n^{\dagger}\left(\zeta_{i}\right) n\left(\zeta_{j}\right)\right| B\right\rangle$ in the canonical basis. The above definition is orientation dependent, and to fix ideas, we have taken

\footnotetext{
${ }^{10}$ The phases $\alpha_{j}^{i}$ also admit a direct geometric interpretation in terms of the framing vector of Ref. [4], but we will not need it here. Notice also that this geometric reconstruction is actually 2 to 1 for the $\mathbb{Z}_{2}$ symmetry discussed earlier, a direct consequence of the usual extra sign appearing when describing a spinor in terms of its flagpole and flag.
} 
a link $i$ with $j$ outgoing from its source node and $k$ outgoing from its target node. We see that analogously to the Wilson loop trace, this gauge-invariant quantity mixes the twist angles $\xi_{i}$ 's and the directions $\zeta_{i}$ 's, as it should; it has the advantage of doing so in a way that a single $\xi_{i}$ enters instead of all of those belonging to the loop. The price to pay is that the definition is not unique; for each link $i$, the gaugeinvariant twist angle depends on a choice of a pair of links $(j k)$ connected to the source and the target of $i$. That is, from the point of view of a triangulation dual to the graph, for each triangle, it depends on a choice of edge. Notice that this is the same redundancy that appears in the construction of Dittrich and Ryan [9], that will be discussed in Sec. IV B. ${ }^{11}$ In terms of these variables, it is also immediate to characterize the shapematching conditions; these are given by

$$
\alpha_{j k}^{i}=\alpha_{l m}^{\tilde{l}} \Leftrightarrow \xi_{j k}^{i}=\xi_{l m}^{i},
$$

namely the matching of the 2D dihedral angles [26] in the first form, or the requirement of a unique gauge-invariant twist angle per link in the second form.

Then, point ii is dealt with precisely by the secondary constraints, which relate the extrinsic curvature $\Xi$ to the SU(2) data. Secondary simplicity constraints have been proposed by Dittrich and Ryan [9,31] as a direct discretization of the Levi-Civitá condition $\omega^{i j}=\omega^{i j}(E)$ but also derived as the stabilization of the primary simplicity constraints in a toy model where a discrete Hamiltonian constraint could be fully solved [10]. As we show below in Sec. IV B, the two definitions coincide and read

$$
\gamma \Xi_{i}-\xi_{j k}^{i}=0
$$

This formula provides the sought relation between the extrinsic curvature and the reduced $\mathrm{SU}(2)$ phase space, and is the state of the art of the understanding of twisted geometries. Notice that these secondary constraints imply the shape-matching conditions (27) $[9,10,31] .{ }^{12}$

To complete this overview of twisted geometries, let us briefly comment on dynamical aspects. The main interest in twisted geometries comes from their relation to spin foam amplitudes and loop quantum gravity, for which they describe the kinematical semiclassical limit on a fixed graph [2]. One can then study the dynamics induced by the quantum theory, and this is a nontrivial task with still a number of open

\footnotetext{
${ }^{11}$ The redundancy can be fixed choosing one representative per link, for instance the average as suggested in Ref. [9]. Once the choice is made, together with the areas $j_{l}$ and the shape variables for each polyhedron ( 2 for tetrahedra, $2 f-6$ for a general polyhedron with $f$ faces), one obtains reduced variables for the gauge-invariant phase space.

${ }^{12}$ An alternative definition of discrete secondary constraints has been proposed in Ref. [32], which does not require the shapematching conditions. It is, however, gauge dependent, and the relation of the extrinsic geometry on the SU(2) data via this alternative construction has not been computed yet, to the best of our knowledge.
}

questions. In particular, a key result [33] is that in the large spin limit of the Engle-Pereira-Rovelli-Livine (EPRL) model on a 4-simplex, the shape-matching conditions are satisfied, and the amplitude is dominated by the Regge action. This is an encouraging result for the model; however, the limiting procedure is delicate to handle on a full triangulation, and it has been argued that only flat solutions are compatible with the saddle point equations of the large spin limit [34]. This has started a debate in the literature on whether nontrivial curvature is properly accounted for, and if not, what the problem is with the model or the limiting procedure. See Refs. [35-37] for some references. Furthermore, the result relies on the special geometric properties of the 4-simplex, and it is not clear how to interpret the most general spin foams that are not dual to a triangulations $[38,39]$. These partial results show the importance of improving our understanding of the dynamics already at the classical level. Actions for twisted geometries and their relations to the Regge action on shell of the shape-matching conditions have been studied in Refs. [9,26,29,40-42], but there is as of yet no clear consensus on the meaning of curvature and dynamics away from the shape-matching subspace nor on the off-shell role of the torsion a priori kinematically present in the theory [13]. An interesting development in this sense is the alternative interpretation of the same phase space in terms of discrete geometries with torsion along the edges proposed in Ref. [43]. Studying the dynamics of the non-shape-matched configurations is also important to understand whether they admit a continuum limit reproducing general relativity or whether the latter property can be satisfied only by the Regge configurations. Lastly, spinors have also found many applications in the study of the quantum dynamics of spin foam models, e.g. Refs. [22,41,44-48].

\section{GEOMETRY OF $\gamma$-NULL AND SIMPLE TWISTORS}

Before studying the action of conformal transformations on twisted geometries, let us look at what the simplicity constraints imply on the usual geometric picture of a twistor. The latter is derived from the incidence relation

$$
\omega^{A}=i X^{A \dot{A}} \bar{\pi}_{\dot{A}},
$$

solving it for $X^{A \dot{A}}(\omega, \pi)$ and interpreting the result as a curve in Minkowski spacetime. ${ }^{13}$ When the twistor is null,

\footnotetext{
${ }^{13}$ In this section, we follow Penrose's notation and use $X^{A \dot{A}}=X^{I}$ to refer to a point or a family of points in Minkowski space as derived from the incidence relation. However, the temptation of identifying its spatial part with the fluxes $\vec{X}$ previously defined should be resisted. As this section shows, when the simplicity constraints are satisfied, $X^{A \dot{A}}$ is proportional to the timelike direction of the constraints, and the fluxes $\vec{X}$ are the spatial parts of the spinor's null poles (the null vector associated with the spinor).
} 
namely $s=0, X^{A \dot{A}}$ is anti-Hermitian, and the solution of (29) is

$$
X^{A \dot{A}}=-\frac{i \omega^{A} \bar{\omega}^{\dot{A}}}{[\pi|\omega\rangle}+b i \pi^{A} \bar{\pi}^{\dot{A}}, \quad b \in \mathbb{R} .
$$

It describes a null ray in the direction of the null pole of $\pi^{A}$, that is $i \pi^{A} \bar{\pi}^{\dot{A}}$, going through a point at a distance $-1 /[\pi|\omega\rangle$ from the origin along the null pole of $\omega^{A}$, that is $i \omega^{A} \bar{\omega}^{\dot{A}}$. The subspace of null twistors is denoted $\mathbb{N}$.

For non-null twistors, the general solution of (29) is instead

$$
X^{A \dot{A}}=-\frac{i \omega^{A} \bar{\omega}^{\dot{A}}}{[\pi|\omega\rangle}+\lambda^{A} \bar{\pi}^{\dot{A}}, \quad \lambda^{A} \in \mathbb{C}^{2} .
$$

This gives a 2-plane in complexified Minkowski spacetime, spanned by the tangent vectors $\lambda^{A} \bar{\pi}^{\dot{A}}$, called the $\alpha$-plane; it is totally null, and its associate bivector is self-dual. Its only intersection with real Minkowski space is along the null ray $i \pi^{A} \bar{\pi}^{\dot{A}}$. However, it is also possible to give an alternative interpretation of non-null twistors in real Minkowski spacetime, by looking at the set of all null twistors $Y^{\alpha}$ incident with $Z^{\alpha}$,

$$
s(Y)=0, \quad \bar{Z} \cdot Y=0 .
$$

This set defines a three-parameter family of null rays associated with the twistor $Z^{\alpha}$. A classic explicit evaluation [7] identifies this family with the Robinson congruence, twisting to the right (righty) or twisting to the left (lefty) according to the sign of $s$, thus giving the name twistor to $Z^{\alpha}$.

Notice that all three geometric pictures (the null ray, $\alpha$ plane, and Robinson congruence) are invariant under a complex rescaling of the twistor,

$$
(\omega, \bar{\pi}) \mapsto \lambda(\omega, \bar{\pi}), \quad \lambda \in \mathbb{C},
$$

and thus more precisely they give a geometric representation of projective twistor space $\mathbb{P \mathbb { T }}$. On the other hand, in the LQG interpretation, the twistor's norms $j$ provide the values of the areas of the triangulation, so it is truly $\mathbb{T}$ and not $\mathbb{P} \mathbb{T}$ that one works with. Conversely, the sign of the helicity does not matter; both righty and lefty twistors give the same geometry, and restriction can be made to positive helicity $j>0$.

Let us now come to the geometric interpretation of the simple twistors solutions of the simplicity constraints (13). From (14), we have that $s=j$, and thus, since we are avoiding the degenerate configurations $j=0$, the simple twistors are not null. As we can always restrict to $j>0$, we see that they describe right-handed Robinson congruences. However, the phase shift

$$
\left(\omega^{A}, \pi_{A}\right) \mapsto\left(\omega^{A}, e^{-i \theta / 2} \pi^{A}\right)
$$

maps a twistor satisfying (14) to a null twistor: the space $\mathbb{N}_{\gamma}$ of solutions to $F_{1}=0$ is isomorphic to $\mathbb{N}$. The isomorphism reduces to the identity at $\theta=0$ or $4 \pi$, that is $\gamma= \pm \infty$. Proper null twistors could only play a role when the simplicity constraints are $L^{i}=0$, that is in the limit where the EPRL model [8] reduces to the Barrett-Crane [49] model. Accordingly, we may refer to twistors satisfying $F_{1}=0$ as " $\gamma$-null" and associate a null ray to them via

$$
X_{\gamma}^{A \dot{A}}=-\frac{i \omega^{A} \bar{\omega}^{\dot{A}}}{j \sqrt{1+\gamma^{2}}}+b i \pi^{A} \bar{\pi}^{\dot{A}}, \quad b \in \mathbb{R} .
$$

Then, the restriction imposed by $F_{2}=0$ is to make the null pole of $\pi^{A}$ proportional to the null pole of $\omega^{A}$. Further, the light ray passes through the point

$$
X_{\gamma}^{I}=-\frac{\sqrt{2}\|\omega\|^{2}}{j \sqrt{1+\gamma^{2}}} N^{I}
$$

consistently with (15), and this fixes

$$
b=-\frac{\|\omega\|^{4}}{j^{3}\left(1+\gamma^{2}\right)^{3 / 2}} .
$$

That is, the condition (15) is picking up a point from the incidence relation, that corresponds to the timelike vector $N^{I}$ specified by the time gauge. Hence, simple twistors describe a subspace of $\mathbb{N}_{\gamma}$ with a timelike vector singled out. Finally, notice also that

$$
\arg \omega^{0}+\arg \omega^{1}+\arg \pi^{0}+\arg \pi^{1}=\theta
$$

so the planes of the spinor's flag are related by a rotation of an angle $\pi-\theta$ in the spacelike plane. Clearly, the construction generalizes from the time gauge $N^{I}=(1,0,0,0)$ considered so far to an arbitrary timelike vector introduced by the simplicity constraints (11).

Summarizing, a simple twistor is a $\gamma$-null twistor with a fixed point along the null ray, so as to align its null poles to the timelike vector provided by the time gauge, and the flag of $\omega$ identifies a spacelike bivector normal to the time direction. The procedure picks up a unique spacelike plane, the one orthogonal to the spatial parts of the null poles, or, equivalently, to $L^{i}$. As discussed below (12) this plane is identified, in covariant terms, by the simple bivector $B \propto\left(\mathbb{1}-\gamma^{\star}\right) J$, and indeed it can be checked that this is the case by simply plugging the solution into the simplicity constraints in the twistor definition of $J$ (3). Hence, the association of a twistor with a light ray becomes secondary upon the imposition of the simplicity constraints, and attention is instead given to this spacelike plane; it is indeed this plane, the building block of twisted geometries, 
that defines the face of a polyhedron, with the norm $j$ fixing its area.

\section{TWISTED GEOMETRIES AND CONFORMAL TRANSFORMATIONS}

The existence of a representation of the conformal group plays an important role in Penrose's vision of twistor space. In fact, the data are used to describe spinning massless particles, which are themselves conformally covariant. The generators acquire a physical interpretation; for instance, $P^{I}$ is the energy momentum of the particle, and $s$ is the helicity. On the other hand, loop quantum gravity suggests an alternative geometric interpretation of twistors as twisted geometries, in which the constraints (8) and (13) plus the closure constraint imposing SU(2) gauge invariance at the nodes reduce twistor space to that of twisted geometry. Therefore, the generators of the conformal group, and the transformations they induce, can acquire a new geometric interpretation. To that end, the action of the group should be compatible with the three sets of constraints defining twisted geometries, that is area matching, simplicity, and closure. A first glance immediately shows that full conformal covariance is broken, as already the (real part of the) area-matching condition imposes $D=\tilde{D}$, which is not preserved by translations and special conformal transformations,

$$
\left\{D, P^{I}\right\}=-P^{I}, \quad\left\{D, C^{I}\right\}=C^{I} .
$$

Hence, these generators do not preserve the constraint surface, and their action does not admit an interpretation in terms of holonomies and fluxes. On the other hand, the dilatation generator commutes with all the constraints; it commutes with $C$, and furthermore

$$
\left\{D, J^{I J}\right\}=0,
$$

and thus it commutes also with the simplicity and closure constraints. The $\operatorname{GL}(2, \mathbb{C})$ subgroup generated by $J^{I J}$ and $D$ is thus compatible with all the constraints.

Unlike in applications of twistor theory to solving the wave equations, the breaking of conformal covariance here is not introduced by the infinity twistor $I_{\alpha \beta}$, which would preserve the isometry group at infinity [Poincaré or (A)dS], but rather by the fixing of the dilatation generators of the two twistors, and preserves instead the subgroup generated by Lorentz transformations and dilatations.

Therefore, orbits of $D$ live in the phase space of loop quantum gravity and can be given a geometric interpretation. On each half-link, say the one associated with the source node, $D$ generates symplectic dilatations on the twistor phase space,

$$
\begin{aligned}
& \exp (\lambda D) \triangleright|\omega\rangle=e^{\lambda / 2}|\omega\rangle, \\
& \exp (\lambda D) \triangleright|\pi\rangle=e^{-\lambda / 2}|\pi\rangle, \quad \lambda \in \mathbb{R} .
\end{aligned}
$$

On each link, we can consider arbitrary real linear combinations of the generators at the source and target, $\mathcal{D}:=\lambda D+\tilde{\lambda} \tilde{D}$. The action of $\mathcal{D}$ on the holonomy-flux algebra gives

$$
\begin{aligned}
\exp (\mathcal{D}) \triangleright \Pi & =\Pi, \quad \exp (\mathcal{D}) \triangleright \tilde{\Pi}=\tilde{\Pi}, \\
\exp (\mathcal{D}) \triangleright h & =\frac{e^{-u / 2}|\tilde{\omega}\rangle\left[\pi\left|-e^{u / 2}\right| \tilde{\pi}\right\rangle[\omega \mid}{\sqrt{[\pi|\omega\rangle} \sqrt{[\tilde{\pi}|\tilde{\omega}\rangle}} \\
& =\cosh (u / 2) h+\sinh (u / 2) \hat{h},
\end{aligned}
$$

where $u:=\lambda+\tilde{\lambda}$ and

$$
\begin{aligned}
\hat{h}_{B}^{A} & :=\frac{\tilde{\omega}^{A} \pi_{B}+\tilde{\pi}^{A} \omega_{B}}{\sqrt{[\pi|\omega\rangle} \sqrt{[\tilde{\pi}|\tilde{\omega}\rangle}} \\
& =-\frac{4}{[\pi|\omega\rangle}(h \Pi)^{A}{ }_{B}, \quad \operatorname{det} \hat{h}=-1 .
\end{aligned}
$$

It is easy to check that the transformed group element still has a unit determinant for all real values of $u$, and thus the orbits span a one-parameter family of embeddings of $\operatorname{SL}(2, \mathbb{C})$ in $\mathbb{T}^{2}$. This symmetry can be understood as follows: when we embed $T^{*} \operatorname{SL}(2, C)$ in twistor space, see (9), the group element is constructed to satisfy

$$
h|\omega\rangle=|\tilde{\omega}\rangle, \quad h|\pi\rangle=|\tilde{\pi}\rangle
$$

and sends an orthogonal basis into another orthogonal one. However, any pair of spinors provides an orthogonal basis provided $[\pi|\omega\rangle \neq 0$, without restriction on the norms, since there is no Lorentz-invariant norm in spinor space. Therefore, one could have equally well demanded parallel transport of the basis up to a rescaling, that is

$$
h^{u}|\omega\rangle=e^{-u / 2}|\tilde{\omega}\rangle, \quad h^{u}|\pi\rangle=e^{u / 2}|\tilde{\pi}\rangle .
$$

The solution to this equation is precisely (42b), and it is clear that any such parametrization would provide an equally good embedding of $T^{*} \operatorname{SL}(2, \mathbb{C})$ in twistor space. The action of $D$ on the phase space corresponds to this symmetry of the symplectic reduction from twistor space.

Next, we would like to understand the geometric meaning of this transformation. First of all, we already know from (40), or equivalently (42a), that the fluxes are unchanged. Indeed, $J^{I J}=\omega^{(A} \pi^{B)}+c c$ is invariant under the symplectic dilatations (41) generated by $D$. However, the Casimirs of the Lorentz generators determine the scales in loop quantum gravity. Therefore, the symplectic dilatations generated by $D$ do not act as geometric dilatations, as they preserve all properties of the three-dimensional 
intrinsic geometry determined by the fluxes: the 2D and 3D angles of the polyhedra, but also their areas and volumes which would rescale under a geometric dilatation.

So what is the effect of (42) on the geometry? Since $\tilde{\Pi}=-h \Pi h^{-1}$, the holonomy is determined by the fluxes up to a rotation and a boost in the direction of $\Pi$. These two variables have been introduced in Ref. [25] as the twist angle $\xi$ and the boost $\Xi$ and form an almost Abelian pair with the Lorentz Casimirs,

$$
\begin{array}{cc}
\{D, \Xi\}=1, \quad & \{D, \xi\}=\gamma, \\
\{s, \Xi\}=0, \quad & \{s, \xi\}=1 .
\end{array}
$$

From the first two brackets, we immediately read the effect of a finite transformation on each link:

$$
e^{\mathcal{D} \triangleright \Xi}=\Xi+u, \quad e^{\mathcal{D} \triangleright \xi}=\xi+\gamma u .
$$

This is the effect of $\mathcal{D}$ on twisted geometries: it shifts the boost and twist angle among adjacent polyhedra.

To interpret these transformations, let us first look at $\xi$ and the reduced action on $T^{*} S U(2)$. We will then discuss the meaning of changing $\Xi$ and prove that the same interpretation holds if we abandon the time gauge and use instead the gauge-invariant parametrization of Dittrich and Ryan [9].

\section{A. Action of $D$ on the reduced phase space and preservation of shapes}

On the SU(2) phase space, the reduced dilatation when the constraints (13) are imposed reads $D=\gamma j$, and its action is

$$
\left\{D, z^{A}\right\}=-\frac{i}{2} \gamma z^{A}, \quad e^{\lambda D} z^{A}=e^{-\frac{i}{2} \gamma \lambda} z^{A} .
$$

Together with the rotation generators $X^{i}, D$ generates a $U(2)$ action on the spinors, already considered in the LQG context for instance in Ref. [41]. As for the full SL(2, C) phase space, the transformation induced by $D$ does not change the fluxes, but only the holonomy,

$$
\begin{aligned}
\exp (\mathcal{D}) & \triangleright \vec{X}=\vec{X}, \quad \exp (\mathcal{D}) \triangleright \overrightarrow{\tilde{X}}=\overrightarrow{\tilde{X}} \\
\exp (\mathcal{D}) \triangleright g & =\frac{e^{\frac{i}{2} \gamma u}|\tilde{z}\rangle\left\langle z\left|+e^{-\frac{i}{2} \gamma u}\right| \tilde{z}\right][z \mid}{\|z\|\|\tilde{z}\|} \\
& =\cos \left(\frac{\gamma}{2} u\right) g+i \sin \left(\frac{\gamma}{2} u\right) \hat{g},
\end{aligned}
$$

where as before $u:=\lambda+\tilde{\lambda}$, and

$$
\hat{g}=\frac{|\tilde{z}\rangle\langle z|-| \tilde{z}][z \mid}{\|z\|\|\tilde{z}\|}, \quad \operatorname{det} \hat{g}=-1,
$$

while $\operatorname{det}(\exp (\mathcal{D}) \triangleright g)=1$. Again, the orbits represent a symmetry of embeddings of $T^{*} \mathrm{SU}(2)$ in $\mathbb{C}^{4}$, which is now represented by defining $g$ as the parallel transport of an orthogonal basis up to a global phase. Using the Hopf sections and the usual twisted geometry parametrization, it is easy to see that the transformation of $g$ is simply the one induced by the transformation of $\xi$ in (47). However, this shift leaves completely invariant the 3D geometry; it only affects the relation between $\xi$ and $\Xi$, that is, the way $g$ is embedded in $T^{*} \operatorname{SL}(2, \mathbb{C})$. This can be hinted at by the limit case $\gamma=0$, for which $g$ is a pure rotation and $D$ vanishes on shell of the simplicity constraints thus leaving $g$ invariant. To make it more explicit, observe that on the full space $\xi$ is given by

$\xi=2 \arg \tilde{z}^{1}-2 \arg z^{1}=2 \arg \tilde{\omega}^{1}-2 \arg \omega^{1}+\gamma \Xi$.

That is, the transformation (47) of $\xi$ under $D$ is the one induced by the transformation of $\Xi$. In other words, $g$ transforms under $D$ because it is not a pure threedimensional holonomy but rather an Ashtekar-Barbero holonomy mixing rotations and boosts.

To complete the argument that the 3D geometry does not change, let us verify the effect of $D$ on the SU(2) gaugeinvariant basis (23). We consider the dilatation generated by an arbitrary linear combination on the graph,

$$
\mathcal{D}_{\Gamma}:=\sum_{l}\left(\lambda_{l} D_{l}+\tilde{\lambda}_{l} \tilde{D}_{l}\right) .
$$

This gives

$$
\begin{aligned}
& \exp \left(\mathcal{D}_{\Gamma}\right) \triangleright\left\langle z_{i} \mid z_{j}\right\rangle=e^{\frac{i}{2} \gamma\left(\lambda_{i}-\lambda_{j}\right)}\left\langle z_{i} \mid z_{j}\right\rangle, \\
& \exp \left(\mathcal{D}_{\Gamma}\right) \triangleright\left[z_{i}\left|z_{j}\right\rangle=e^{-\frac{i}{2} \gamma\left(\lambda_{j}+\lambda_{i}\right)}\left[z_{i}\left|z_{j}\right\rangle .\right.\right.
\end{aligned}
$$

From these, we deduce that for a finite dilatation areas and 3D dihedral angles do not change, whereas the spinor phases transform as $\delta \alpha_{j}^{i}=-\gamma \lambda_{i}$. Notice that the transformation is independent of the lower label $j$ in $\alpha_{j}^{i}$. As a consequence,

$$
\delta \alpha_{j k}^{i}=0, \quad \delta \xi_{j k}^{i}=\gamma\left(\lambda_{i}+\tilde{\lambda}_{i}\right) .
$$

The 2D dihedral angles $\alpha_{j k}^{i}$ are unchanged, consistently with the fact that dilatations do not affect the fluxes. The gauge-invariant twist angle $\xi_{j k}^{i}$ is affected, consistently with the holonomy transformation (49b). However, it is changed in a way that is independent of the lower labels, and thus the transformation cannot change whether the shapes match or not. Furthermore, from (47) and (54), we see that the transformation preserves the secondary constraints (28).

We conclude that the action of $\mathcal{D}$ does not change the intrinsic 3D geometry. In particular, it does not break the embedding provided by the secondary constraints, nor the shape-matching conditions. These properties make it 
compatible also as a transformation on the Regge phase space.

\section{B. Unfixing the time gauge}

The way we parametrized the covariant twisted geometries, in particular the boost dihedral angle $\Xi$, uses explicitly the time gauge. This is simply a convenience to use the primary simplicity constraints in the linear form (12), and all results can be shown to be covariant under the change of gauge (e.g. Ref. [15]). However, one can also consider a formulation of the theory in terms of purely gauge-invariant quantities and quadratic simplicity constraints. In this setting, we cannot use a definition such as (21) to define the extrinsic curvature, but we can define it via the edge vectors of the triangulation. This construction was proposed and carried out in Ref. [9] for Euclidean signature. Here, we extend it to the Lorentzian signature, and because the construction is based on the self-dual/antiself-dual splitting, it means dealing with complex vectors instead of real vectors. We then show that the resulting angle is transformed by $D$ in exactly the same way as $\Xi$. In doing so, we work out the explicit relation between the dihedral angle of Ref. [9] and $\Xi$ and find out that the secondary simplicity constraints proposed in Ref. [9] are precisely the ones arising in Ref. [10].

To fix ideas, consider again an oriented graph $i$ dual to a triangulation, with $j$ outgoing from its source node and $k$ outgoing from its target node. We define the anti-self-dual edge vectors

$$
\vec{Y}_{i j}:=\vec{\Pi}_{i} \times \vec{\Pi}_{j} .
$$

The same edge is shared by the adjacent tetrahedron, with associated edge vector $\vec{Y}_{i k}$. The fact that there are two or more independent edge vectors associated with the same edge is just another way of seeing the familiar shape mismatching of twisted geometries. Nonetheless, we can define a (complex) angle among these two vectors as follows:

$$
\cosh \theta_{j k}^{i}=\frac{\vec{Y}_{\tilde{\imath} k} \cdot h_{i} \triangleright \vec{Y}_{i j}}{\sqrt{\vec{Y}_{\tilde{\imath} k}^{2}} \sqrt{\vec{Y}_{i j}^{2}}}
$$

This formula adapts the definition of Dittrich and Ryan [9] to the Lorentzian signature, using complex angles as in the setup of Sorkin for Lorentzian triangulations [50]. Notice that this definition associates three angles to each face (in the case of a triangulation, or as many as the valence of the face in the case of a more general cellular decomposition). The shape mismatch is then characterized by the fact that these angles take different values, and the shape-matching conditions read $\theta_{j k}^{i}=\theta_{l m}^{i}$. We thus have exactly the same redundancy and structure of the shape-matching conditions as we do when using reduced $\mathrm{SU}(2)$ variables and twisted geometries; see (27).

To study the action of $\mathcal{D}$ on these angles, we first have to parametrize them in terms of Lorentzian spinors. Nicely, one obtains a simple expression with only two terms:

$$
\cosh \theta_{j k}^{i}=\frac{1}{2} \frac{\left[\omega _ { i } | \pi _ { j } \rangle \left[\omega _ { i } | \omega _ { j } \rangle \left[\tilde { \pi } _ { i } | \omega _ { k } \rangle \left[\tilde{\pi}_{i}\left|\pi_{k}\right\rangle+\left[\pi _ { i } | \omega _ { j } \rangle \left[\pi _ { i } | \pi _ { j } \rangle \left[\tilde { \omega } _ { i } | \pi _ { k } \rangle \left[\tilde{\omega}_{i}\left|\omega_{k}\right\rangle\right.\right.\right.\right.\right.\right.\right.\right.}{\sqrt{\left[\omega _ { i } | \omega _ { j } \rangle \left[\pi _ { i } | \pi _ { j } \rangle \left[\omega _ { i } | \pi _ { j } \rangle \left[\pi_{i}\left|\omega_{j}\right\rangle\right.\right.\right.\right.} \sqrt{\left[\omega _ { k } | \tilde { \omega } _ { i } \rangle \left[\pi _ { k } | \tilde { \pi } _ { i } \rangle \left[\omega _ { k } | \tilde { \pi } _ { i } \rangle \left[\pi_{k}\left|\tilde{\omega}_{i}\right\rangle\right.\right.\right.\right.}} .
$$

The action of the graph generator $\mathcal{D}$ can then be easily computed from (41) to give

$$
e^{\mathcal{D}} \triangleright \cosh \theta_{j k}^{i}=\cosh \left(\theta_{j k}^{i}+u_{i}\right) .
$$

Here, we used the fact that (57) with a relative minus sign gives $\sinh \theta_{j k}^{i}$. Remarkably, we have found precisely the same result as for $\Xi_{i}$ [see (47)]: the dilatation shifts the extrinsic curvature. Again, the preservation of the shapes follows from the fact that the change on $\theta_{j k}^{i}$ is independent of the edge used to define it.

The result can be made more transparent if we reintroduce the time gauge and write (57) on shell of the simplicity constraints. A straightforward calculation then gives

$$
\cosh \theta_{j k}^{i}=\cosh \left(\Xi_{i}+i\left(\gamma \Xi_{i}-\xi_{j k}^{i}\right)\right)
$$

This formula gives the relation in the time gauge between the Dittrich-Ryan dihedral angle $\theta_{j k}^{i}[\operatorname{SL}(2, \mathbb{C})$ gauge invariant but edge dependent] and the twisted geometry dihedral angle $\Xi_{i}$ [edge independent but not $\operatorname{SL}(2, \mathbb{C})$ gauge invariant, only $\mathrm{SU}(2)]$. Using it, we can also prove that the secondary simplicity constraints defined in Ref. [9] as

$$
\theta_{j k}^{i}=\bar{\theta}_{j k}^{i}
$$

amount precisely to (28), which were derived dynamically through a toy model [10]. And finally, Eq. (58) can be derived directly from (47), and the fact already remarked that the secondary constraints are preserved.

\section{Some dynamical considerations}

We have seen that the dilatation generator acts as a translation on the boost between two polyhedra, be it defined via the time gauge as in (21) or via the edge vectors as in (56). In a spacetime picture à la Regge, this plays the role of the dihedral angle providing the extrinsic geometry 
of the embedding in a Lorentzian discrete metric. Therefore, the rescaling (47) generated by $D$ changes the extrinsic geometry, and as the 3D geometry is unchanged, it will affect the spacetime geometry. This suggests that $D$ could play a role in dynamical models, something that we leave for future work. In fact, not all orbits of $D$ are compatible with a discrete dynamics à la Regge. Restrictions for instance arise from the requirement of discretizing spacetime with flat 4-simplices. As a simple examples of this, we can consider a triangulation with 5-tetrahedra bounding a 4-simplex; in this case, it is clear that imposing flatness in the bulk kills any action of $D$, since the four-dimensional dihedral angles are then uniquely determined by the areas (up to special configurations). ${ }^{14}$ Or, consider a triangulation with three 4-simplices sharing a face in the bulk, so that fourdimensional curvature is allowed via the deficit angle associated to the bulk face. By definition of the deficit angle, different curvatures can be distinguished by the different values of the boundary dihedral angles. Thus, it is now possible to vary the boundary dihedral angles at fixed areas, using $\mathcal{D}$, and induce in this way a different curvature at the bulk face, giving a clear connection between $\mathcal{D}$ and dynamical aspects. Notice, however, that the admissible orbits in this case are spanned by a single-parameter family and not by independent shifts.

Generally speaking, a relation between dilatations and dynamics is to be expected, since we know that in the continuum theory the generator of symplectic dilatations in the phase space of triads and connections appears in the Hamiltonian constraint [53]. ${ }^{15}$ It is then interesting to also consider a discretized version of the connection-triad symplectic dilatation and compare its action with the one of $D$, the twistor symplectic dilatation. This is what we do in the next section.

\section{HOLONOMY-FLUX SYMPLECTIC DILATATIONS}

On the reduced phase space $T^{*} \mathrm{SU}(2)$, the twistor symplectic dilatation $D \approx \gamma j$ preserves areas and volumes. The scale invariance of the transformation is also clear if we look at the continuum limit of $D$; this gives the norm of the

\footnotetext{
${ }^{14}$ The action of $D$ would be admissible if one works with a 4-simplex of constant curvature, since we know from the Schläfli identity that on a curved 4-simplex a shift of the dihedral angles at constant areas and 3D geometry induces a shift of the 4-volume, $\sum_{l} A_{l} \delta \Xi_{l}=3 \kappa \delta V$, where $\kappa$ is the 4-simplex curvature. However, for this interpretation to make sense, the boundary tetrahedra and triangles should be constantly curved themselves, and this is not the usual interpretation of twisted geometries. Since curved 4-simplices are expected to arise in dynamical loop quantum gravity models with a cosmological constant, e.g. Refs. [51,52], it may be that $D$ could play a role in dynamical models with a cosmological constant.

${ }^{15}$ More recently, its role has been put to evidence for the dynamics of cosmological models with nonzero $\Lambda$ [54].
}

Ashtekar flux $E^{i}$, which commutes with all fluxes and thus the full three-dimensional metric. At the continuum level, a symplectic dilatation in the phase space of AshtekarBarbero variables is generated by

$$
\begin{aligned}
W & =E_{i}^{a} A_{a}^{i}, \\
\exp (\lambda W) \triangleright A_{a}^{i} & =e^{-\lambda} A_{a}^{i}, \\
\exp (\lambda W) \triangleright E_{a}^{i} & =e^{\lambda} E_{a}^{i},
\end{aligned}
$$

where we used the canonical algebra satisfied by the connection and the triad. ${ }^{16}$ In this section, we only deal with a single copy of the cotangent bundle, so we do not need the label $i$ for the links, and we can safely use it for the $\mathfrak{g} \mathfrak{t}(2)$ indices.

Since the phase space $T^{*} \mathrm{SU}(2)$ has compact directions, there is no dilatation generator in the sense of (61). However, being ultimately interested in the continuum limit of the theory, it makes sense to consider a discretization of $W$ acting on $T^{*} \mathrm{SU}(2)$. A simple choice is given by the Lie derivative of the group character,

$$
W=2 \mathcal{L}_{X} \chi^{\left(\frac{1}{2}\right)}(g)=-2 \operatorname{Tr}(g X),
$$

which reduces to (61) in the continuum limit, if we denote $g \simeq \mathbb{1}+A, A:=A_{a}^{i} \tau_{i} \ell^{a}, X^{i} \simeq E_{i}, E_{i}:=E_{i}^{a} s_{a}$, with $\ell^{a}$ the coordinate tangent to the link at its source and $s_{a}$ the coordinate area 2-form of its dual surface. Let us study the transformations induced by (62) on the holonomy-flux variables. At the infinitesimal level, we have

$$
\begin{aligned}
\{W, g\} & =\mathbb{1}-\frac{\operatorname{Tr} g}{2} g, \\
\left\{W, X^{i}\right\} & =\frac{1}{2} \operatorname{Tr} g X^{i}-\epsilon^{i j k} X^{j} \operatorname{Tr}\left(\tau^{k} g\right) .
\end{aligned}
$$

To compute the finite action, it is easier to use the spinorial parametrization (19). Interestingly, $W$ acts as a boost in mixing the spinors,

$$
e^{\lambda W} \triangleright\left(\begin{array}{c}
z^{A} \\
\tilde{z}^{A}
\end{array}\right)=\left(\begin{array}{cc}
\cosh \lambda / 2 & \sinh \lambda / 2 \\
\sinh \lambda / 2 & \cosh \lambda / 2
\end{array}\right)\left(\begin{array}{c}
z^{A} \\
\tilde{z}^{A}
\end{array}\right) .
$$

Using (64), it is not hard to show that

\footnotetext{
${ }^{16} \mathrm{Next}$ to this kinematical dilatation, one could also consider a more "dynamical" dilatation, where the splitting of the AshtekarBarbero connection into a Levi-Civitá part plus extrinsic curvature is taken into account. This would lead then to a nonlinear transformation,

$$
A_{a}^{\prime i}=\Omega^{-2} A_{a}^{i}+\left(1-\Omega^{-2}\right) \Gamma_{a}^{i}(E)+2 \epsilon^{i j k} E_{a}^{i} E_{k}^{b} \partial_{b} \ln \Omega,
$$

which was considered for instance in Ref. [55].
} 


$$
\begin{aligned}
g_{W} & :=e^{\lambda W} \triangleright g \\
& =\frac{1}{\cosh \lambda+\frac{\operatorname{Tr} g}{2} \sinh \lambda}\left[\cosh ^{2} \frac{\lambda}{2} g+\sinh ^{2} \frac{\lambda}{2} g^{-1}+\sinh \lambda \mathbb{\pi}\right],
\end{aligned}
$$

$$
\begin{aligned}
X_{W}^{i}:= & e^{\lambda W} \triangleright X^{i} \\
= & X^{i}+\left(\sinh ^{2} \frac{\lambda}{2}+\frac{X^{2} \operatorname{Tr} g}{2\left(X^{2}-X \cdot \tilde{X}\right)} \sinh \lambda\right) \\
& \times\left(X^{i}-\tilde{X}^{i}\right)-\frac{\operatorname{Tr}(g X)}{X^{2}-X \cdot \tilde{X}} \sinh \lambda \epsilon^{i j k} X^{j} \tilde{X}^{k} .
\end{aligned}
$$

The transformations are nonlinear, ${ }^{17}$ as expected, and cannot be visualized as symplectic dilatations in the continuum sense (61). Nonetheless, they have some interesting properties. First of all, they preserve the Poisson structure. To see that, let us rewrite them in a compact way as follows,

$$
g_{W}=S^{\dagger} g S^{\dagger}, \quad X_{W}:=X_{W}^{i} \tau_{i}=\mathcal{N}\left(S X S^{\dagger}\right),
$$

where

$$
\begin{aligned}
\mathcal{N} & =\cosh \lambda+\frac{\operatorname{Tr} g}{2} \sinh \lambda \\
S & =\frac{1}{\sqrt{\mathcal{N}}}\left(\cosh \frac{\lambda}{2} \mathbb{1}+\sinh \frac{\lambda}{2} g\right) .
\end{aligned}
$$

It is easy to show that $S \in \mathrm{SU}(2)$, and thus also $g_{W}$. Hence, the transformation preserves the polarization. To verify preservation of the Poisson brackets, again it is easiest to use spinors; Eq. (64) is easily seen to preserve the spinorial brackets, and the area-matching constraint is changed, but only by a nonzero multiplicative factor, $e^{\lambda W} \triangleright C=\mathcal{N} C$. Hence, the symplectic reduction is still $T^{*} \mathrm{SU}(2)$.

Furthermore, it is easy to check that the transformations (65) have the right continuum limit; for $g \approx \mathbb{1}+A$, we get

$$
g_{W} \simeq \mathbb{1}+e^{-\lambda} A, \quad X_{W}^{i} \simeq e^{\lambda} X_{i} .
$$

This means that the holonomy-flux "dilatation" operator captures in the continuum limit the properties of a connection-triad dilatation: rescaling of areas and volumes with preservation of angles and rescaling of the connection.

\footnotetext{
${ }^{17}$ The nonlinearity of the transformations $(65)$ can be organized in powers of $\operatorname{Tr} g$ and $\operatorname{Tr}(g X)$, since $2\left(X^{2}-X \cdot \tilde{X}\right)=$ $j^{2} \operatorname{Tr}^{2} g+4 \operatorname{Tr}^{2}(g X)$. It is intriguing that similar nonlinear structures with class-function factors $\operatorname{Tr} g$ and $\operatorname{Tr}(g X)$ appear in the construction of a phase space for curved simplices [56,57], related in turn to Poisson-Lie groups and Hopf algebras. However, we do not know at this stage if $W$ has any specific role to play there.
}

Furthermore, we will see below that in the same continuum limit, the covariant version of $W$ reproduces the linear shifts of $\Xi$ generated by $D$.

On the other hand, let us stress that, unlike $D$, this action is not generally compatible with the discrete geometric interpretation, since the closure condition is disrupted,

$$
e^{\lambda W} \triangleright \sum_{l \in n} \vec{X}_{l}=\sum_{l \in n} \vec{X}_{W l} \neq 0,
$$

a breaking that occurs because of the nonlinearity of the transformation. At leading order in the continuum limit, the closure defect can be approximated with $\sum_{l \in n} e^{\lambda_{l}} \vec{X}_{l}$, and thus in this limit, the symmetry is recovered at least for global transformations. ${ }^{18}$

The situation can be compared with Regge calculus. Since the fundamental variables are now lengths instead of areas and angles as in twisted geometries, local conformal transformations are defined rescaling the edge lengths at both ends [6], $\delta \ell_{x y}=\left(\lambda_{x}+\lambda_{y}\right) \ell_{x y}$. This ensures that the triangulation is not disrupted; however, the volumes are changed by a scaling, and the angles are not invariant. Furthermore, it is not possible to extend this transformation to a finite group action, because the composition of two finite transformations generically breaks the triangle inequalities. Nonetheless, this definition has useful applications to discrete Ricci flow [58,59] and quantum Regge calculus [60].

We conclude that, while $W$ provides an interesting nonlinear definition of dilations in the compact phase space $T^{*} \mathrm{SU}(2)$, with a well-behaved continuum limit, its generic incompatibility with the closure constraint hinders direct applications to classical dynamical models on a fixed graph. On the other hand, it could intervene interestingly in situations where the closure constraint is relaxed, such as in coarse graining $[61,62]$ or in some spin foam models $[35,36]$.

\section{A. Transformations on twisted geometries}

For completeness, we report the action of $W$ on the various twisted geometry variables,

$$
\begin{gathered}
e^{\lambda W} \triangleright j=j\left(\cosh \lambda+\frac{\operatorname{Tr} g}{2} \sinh \lambda\right), \\
e^{\lambda W} \triangleright \zeta=\frac{\cosh \frac{\lambda}{2} \sqrt{1+|\tilde{\zeta}|^{2}} \zeta+\sinh \frac{\lambda}{2} \sqrt{1+|\zeta|^{2}} e^{\frac{i}{2} \xi} \tilde{\zeta}}{\cosh \frac{\lambda}{2} \sqrt{1+|\tilde{\zeta}|^{2}}+\sinh \frac{\lambda}{2} \sqrt{1+|\zeta|^{2}} e^{\frac{i}{2} \xi}},
\end{gathered}
$$

\footnotetext{
${ }^{18}$ For graphs with $L \geq 3 N$, it may also be possible to find nontrivial sets of $\lambda_{l}$ for which the closure defects vanish everywhere.
} 


$$
e^{\lambda W} \triangleright \xi=2 \arctan \left[\frac{\sqrt{1+|\zeta|^{2}} \sqrt{1+|\tilde{\zeta}|^{2}} \sin \xi / 2}{\sqrt{1+|\zeta|^{2}} \sqrt{1+|\tilde{\zeta}|^{2}} \cosh \lambda \cos \xi / 2+\left(1+\left(|\zeta|^{2}+|\tilde{\zeta}|^{2}\right) / 2\right) \sinh \lambda}\right] .
$$

In particular, the last one shows the strong departure from the simple action of $D .{ }^{19}$ In comparing the two operators, it is somewhat curious to point out that this complicated action stems from what looks like a rather minimal nonlinear operator: in the twisted geometries parametrization, we can write

$$
W=-2 j \partial_{\xi} \operatorname{Tr} g,
$$

whereas we have already seen on the reduced space that $D=\gamma j$.

On the gauge-invariant variables, we have

$$
e^{\lambda W_{i}} \triangleright\langle i \mid j\rangle=\cosh \frac{\lambda}{2}\langle i \mid j\rangle+\sinh \frac{\lambda}{2}\langle\tilde{\imath} \mid j\rangle,
$$

from which we deduce that

$$
e^{\lambda_{j} W_{j}} \triangleright \xi_{j k}^{i} \neq e^{\lambda_{k} W_{k}} \triangleright \xi_{j k}^{i},
$$

so a general transformation generated by $W$ will also disrupt the matching of shapes.

We can also reproduce the continuum limit results. In the parametrization of twisted geometries, the continuum limit used in (68) reads $\tilde{\zeta} \simeq \zeta+\delta \tilde{\zeta}$ and $\xi \simeq \delta \xi$, and $A=\delta \tilde{n} n^{-1}+\delta \xi n \tau_{3} n^{-1}$. In this limit, Eqs. (68) and (72) reduce to

$$
\begin{array}{ll}
e^{\lambda W} \triangleright j \simeq e^{\lambda} j, & e^{\lambda W} \triangleright \zeta \simeq \zeta+\frac{1}{2}\left(1-e^{-\lambda}\right) \delta \tilde{\zeta}, \\
e^{\lambda W_{D}} \simeq \xi \simeq e^{-\lambda} \delta \xi, \quad e^{\lambda W_{i}} \triangleright\langle i \mid j\rangle \simeq e^{\lambda}\langle i \mid j\rangle .
\end{array}
$$

We recover in this way the preservation of angles and shape-matching conditions as well as the rescaling of the part of the connection carried by $\xi$.

\section{B. Covariant action}

Finally, let us consider a covariant version of $W$ and study its action on the extrinsic geometry $\Xi$. At first sight, we could simply take

$$
W^{\prime}:=-4 \operatorname{Tr}(h \Pi)=\pi \tilde{\omega}+\omega \tilde{\pi} .
$$

However, on shell of the simplicity constraints, this mixes $g$ and $\hat{g}$ :

\footnotetext{
${ }^{19}$ Notice that for $\sinh ^{2} \frac{\lambda}{2}=|\zeta|^{-2}$ the transformation on $\zeta$ reduces to a conformal transformation of $\mathbb{C P}^{1}$ parametrized by $\tilde{\zeta}$ and $\xi$. However, it is not possible to fix $\lambda$ so as to have a conformal transformation for both $\zeta$ and $\tilde{\zeta}$.
}

$$
\begin{aligned}
W^{\prime} \approx & -2(1-i \gamma)\left[\cosh \left((1+i \gamma) \frac{\Xi}{2}\right) \operatorname{Tr}(g X)\right. \\
& \left.+\sinh \left((1+i \gamma) \frac{\Xi}{2}\right) \operatorname{Tr}(\hat{g} X)\right] .
\end{aligned}
$$

The mixing can be easily avoided considering also

$$
\hat{W}^{\prime}:=-4 \operatorname{Tr}(\hat{h} \Pi) \equiv[\pi|\omega\rangle \operatorname{Tr} h=\pi \tilde{\omega}-\omega \tilde{\pi}
$$

and taking the following linear combination:

$$
\begin{aligned}
\mathcal{W} & :=\frac{\cosh \left((1+i \gamma) \frac{\Xi}{2}\right) W^{\prime}-\sinh \left((1+i \gamma) \frac{\Xi}{2}\right) \hat{W}^{\prime}}{(1-i \gamma) \cosh [(1+i \gamma) \Xi]} \\
& \equiv \frac{e^{-(1+i \gamma) \overline{\frac{\Xi}{2}}} \pi \tilde{\omega}+e^{(1+i \gamma) \overline{\frac{\Xi}{2}}} \omega \tilde{\pi}}{(1-i \gamma) \cosh [(1+i \gamma) \Xi]} \approx-2 \operatorname{Tr}(g X) \equiv W
\end{aligned}
$$

Equipped with this covariant version of the generator of connection-triad symplectic dilatations, we can evaluate its action on $\Xi$, for which we obtain

$$
\begin{aligned}
\{\mathcal{W}, \Xi\}= & \frac{1}{(1-i \gamma) \cosh [(1+i \gamma) \Xi]} \\
& \times\left(e^{-(1+i \gamma) \frac{\bar{\Sigma}}{2}} \frac{\langle\omega \mid \tilde{\omega}\rangle}{\|\omega\|^{2}}+e^{(1+i \gamma) \frac{\Xi}{2}} \frac{\langle\tilde{\omega} \mid \omega\rangle}{\|\tilde{\omega}\|^{2}}\right) \\
\approx & \frac{2}{1-i \gamma} \sqrt{\frac{1-N \cdot \tilde{N}}{2}}\left[\cos \left(\frac{\xi}{2}+\arg (1+\tilde{\zeta} \bar{\zeta})\right)\right. \\
& \left.-i \sin \left(\frac{\xi}{2}+\arg (1+\tilde{\zeta} \bar{\zeta})\right) \tanh ((1+i \gamma) \Xi)\right] .
\end{aligned}
$$

Expanding at first order in the continuum limit $h=\mathbb{1}+o(A)$, we get

$$
\{\mathcal{W}, \Xi\} \simeq \frac{2}{1-i \gamma} .
$$

Up to a numerical factor, which is due to the fact that we started with $\Pi$ instead of the rotation generator $L=\Pi^{i}+\bar{\Pi}^{i}$, we have reproduced the translation transformation generated by $D$.

To summarize, comparing the action of $\mathcal{W}$ with $D$, we see that at leading order in the continuum limit (68), the action of $\mathcal{W}$ changes areas and volumes, preserves the 2D and 3D angles, and reproduces the shift in extrinsic curvature caused by $D$. This is an interesting property; however, as already stated, care is needed in the use of $\mathcal{W}$ as 
the shifts in the areas are in general incompatible with the closure constraint.

\section{CONCLUSIONS}

The main goal of the paper was to study the action of the group of conformal isometries of Minkowski space on the phase space of loop quantum gravity on a fixed graph, in its description in terms of twisted geometries. To do so, we used the twistorial parametrization and the Hamiltonian action of $\mathrm{SU}(2,2)$ on twistor space. We showed that translations and special conformal transformations are not compatible with the various constraints reducing twistor space to twisted geometries but that the dilatation generator is. The origin of this compatibility is to be found in a previously unstudied symmetry of embeddings of $\operatorname{SL}(2, \mathbb{C})$ in twistor space, or equivalently of $T^{*} \mathrm{SU}(2)$ in $\mathbb{C}^{4}$. The associated orbits have an interesting geometric meaning. They change the extrinsic geometry by a linear shift, described by boosts among adjacent polyhedra, as well as the embedding of the SU(2) holonomy in the covariant phase space, an embedding handled at the continuum level by the Barbero-Immirzi canonical transformation and imposition of secondary constraints.

In doing so, we highlighted the role of the diagonal simplicity and area matching in breaking full conformal invariance and discussed the geometric meaning of the various constraints from the viewpoint of twistor theory, showing an isomorphism with null twistors, and how the simplicity constraints identify a spacelike plane that is then used for the geometric reconstruction. Our analysis can be extended in many directions. First, investigating the dynamical applications of $D$, either in a conventional setting with flat 4-simplices, see also Ref. [54] on this, or in an alternative setting with curved 4-simplices, as advocated for instance in Ref. [63]. In the latter case, while the twistorial description of the conformal group immediately applies, the usual phase space description in terms of twisted geometries has to be changed, presumably along the lines investigated in Refs. [56,57]. Similarly, the geometric meaning and possible application of $W$ deserves further study. Since symplectic dilatations generate squeezed coherent states for a particle on a line, it would be interesting to see whether $W$ can be used to construct interesting squeezed spin networks, a topic of recent interest in the community [64-66].

Finally, we hope to come back in future research to the more formal aspect of our motivations and use twistor methods to study conformal spin networks and their applications to loop quantum gravity. ${ }^{20}$

\footnotetext{
${ }^{20}$ Here, we have in mind $\mathrm{SU}(2,2)$ spin networks, as mentioned in the Introduction. A relation between spin networks and the infinite-dimensional group of conformal transformations in two dimensions has been hinted at recently in Refs. [67-69].
}

\section{ACKNOWLEDGMENTS}

We thank Bianca Dittrich, Tim Koslowski, Laurent Freidel, Etera Livine, and Wolfgang Wieland for discussions and Laurent Freidel for sharing a draft of Ref. [54]. M. L. acknowledges Grant No. 266101 from the Academy of Finland.

\section{APPENDIX A: ALGEBRA CONVENTIONS}

We take the generators of $\mathfrak{s} \mathfrak{t}(2,2)$ to satisfy

$$
\left\{\boldsymbol{M}^{a b}, \boldsymbol{M}^{c d}\right\}=\eta^{a c} \boldsymbol{M}^{b d}-\eta^{a d} \boldsymbol{M}^{b c}+\eta^{b d} \boldsymbol{M}^{a c}-\eta^{b c} \boldsymbol{M}^{a d},
$$

with $a=0$ to 5 , and $\eta^{a b}=\operatorname{diag}(-++++-)$. We further fix conventions $\epsilon_{012345}=\epsilon^{012345}=\epsilon^{0123}=1, \quad \epsilon^{I J K L}=$ $\epsilon^{I J K L 45}$. To highlight the various subalgebras of $\mathfrak{g} \mathfrak{t}(2,2)$, we introduce the following notation,

$$
\begin{aligned}
& J^{I J}=M^{I J}, \quad P^{I}=\frac{1}{\sqrt{2}}\left(M^{I 5}+M^{I 4}\right), \\
& C^{I}=\frac{1}{\sqrt{2}}\left(M^{I 5}-M^{I 4}\right), \quad D=-M^{45},
\end{aligned}
$$

where $I=0$ to 3 . The $J$ generate the Lorentz subalgebra and can be further decomposed as

$$
\begin{aligned}
& K^{i}=L^{0 i}, \quad L^{i}=-\frac{1}{2} \epsilon_{j k}^{i} L^{j k}, \\
& \Pi^{i}=i J_{-}^{0 i}=\frac{1}{2}\left(L^{i}+i K^{i}\right) .
\end{aligned}
$$

In terms of this decomposition, the Poisson brackets (A1) read

$$
\begin{aligned}
\left\{L^{i}, L^{j}\right\} & =-\epsilon^{i j k} L^{k}, \\
\left\{L^{i}, K^{j}\right\} & =-\epsilon^{i j k} K^{k}, \\
\left\{K^{i}, K^{j}\right\} & =\epsilon^{i j k} L^{k}, \\
\left\{P^{I}, J^{J K}\right\} & =-\eta^{I J} P^{K}+\eta^{I K} P^{J}, \\
\left\{C^{I}, J^{J K}\right\} & =-\eta^{I J} C^{K}+\eta^{I K} C^{J}, \\
\left\{C^{I}, P^{J}\right\} & =\eta^{I J} D-J^{I J}, \\
\left\{D, P^{I}\right\} & =-P^{I}, \\
\left\{D, C^{I}\right\} & =C^{I} .
\end{aligned}
$$

In the main text, we refer to the three Casimir invariants of $\mathfrak{g} \mathfrak{u}(2,2)$. These are given by

$$
\begin{gathered}
\mathcal{C}^{(2)}=M_{a b} M^{a b}=2 C_{1}-2 D^{2}-4 P \cdot C, \\
\mathcal{C}^{(3)}=\frac{1}{8} \epsilon_{a b c d e f} M^{a b} M^{c d} M^{e f}=3 D C_{2}-6 W \cdot C,
\end{gathered}
$$




$$
\begin{aligned}
\mathcal{C}^{(4)}= & M_{a b} M^{b c} M_{c d} M^{d a} \\
= & 2 C_{1}^{2}+C_{2}^{2}+2 D^{4}+8 J_{I J} J^{J K} P^{I} C_{K}+8 D J_{I J} P^{I} C^{J} \\
& +8 D^{2} P \cdot C+4 P^{2} C^{2}+4(P \cdot C)^{2},
\end{aligned}
$$

where

$$
\begin{aligned}
& C_{1}=\frac{1}{2} J_{I J} J^{I J}=L^{2}-K^{2}=2\left(\Pi^{2}+\bar{\Pi}^{2}\right), \\
& C_{2}=\frac{1}{2}(\star J)_{I J} J^{I J}=2 K \cdot L=2 i\left(\Pi^{2}-\bar{\Pi}^{2}\right)
\end{aligned}
$$

are the Lorentz Casimirs and

$$
W_{I}=\frac{1}{2} \epsilon_{I J K L} M^{J K} P^{L}
$$

is the Pauli-Lubanski vector, which satisfies $W_{I}=-s P_{I}$ for a massless particle. In deriving $C^{(4)}$, we used the identities

$$
\begin{aligned}
J_{I J} J^{J K} P^{I} C_{K} & =-\frac{1}{2} \epsilon_{I J K L} J^{I J} W^{K} C^{L}-\frac{1}{2} J_{I J} J^{I J} P \cdot C, \\
J_{I J} J^{J K} J_{K L} J^{L I} & =2 C_{1}{ }^{2}+C_{2}{ }^{2} .
\end{aligned}
$$

\section{APPENDIX B: SPINORIAL NOTATION}

Throughout the paper, we use regularly Penrose's abstract index convention and spinor formalism. The key difference between our conventions and those more commonly used in the twistor literature (e.g. Ref. [70]) is the metric signature, which we take to be mostly plus. Then, the abstract index map $I=A \dot{A}$ can be realized in terms of anti-Hermitian matrices. That is,
$X^{A \dot{A}}=\frac{i}{\sqrt{2}} \sigma_{I}^{A \dot{A}} X^{I}=\frac{i}{\sqrt{2}}\left(\begin{array}{cc}X^{0}+X^{3} & X^{1}-i X^{2} \\ X^{1}+i X^{2} & X^{0}-X^{3}\end{array}\right)$.

Spinorial indices are raised and lowered with the antisymmetric $\epsilon_{A B}$ symbol, $\omega_{A}=\omega^{B} \epsilon_{B A}$, with $\epsilon_{01}=1$. We further use the following ket notation to eliminate explicit indices in most formulas:

$$
\begin{gathered}
|\omega\rangle=\omega^{A}, \\
\langle\omega|=| \omega\rangle^{\dagger}=\delta_{A \dot{A}} \bar{\omega}^{\dot{A}}, \\
\|\omega\|^{2}=\langle\omega \mid \omega\rangle, \\
\mid \omega]:=-\epsilon|\bar{\omega}\rangle=-\delta^{A \dot{B}} \epsilon_{\dot{B} \dot{A}} \bar{\omega}^{\dot{A}}, \\
{\left[\omega \mid=\omega_{A}=\omega^{B} \epsilon_{B A},\right.} \\
{\left[\pi|\omega\rangle=\epsilon_{A B} \pi^{A} \omega^{B} .\right.}
\end{gathered}
$$

Finally, we take self-dual projectors $P_{ \pm K L}^{I J}=\frac{1}{2}(\mathbb{1} \mp i \star)$, satisfying $\star P_{ \pm}= \pm i P_{ \pm}$. Following Penrose, we call selfdual (or right-handed) the positive eigenspace, and map it to dotted indices, and anti-self-dual (or left-handed) the negative one, and map it to undotted indices. Accordingly, for a bivector, we have

$$
\begin{aligned}
F^{I J} & =P_{-K L}^{I J} F^{K L}+P_{+K L}^{I J} F^{K L} \\
& =F^{A B \dot{A} \dot{B}} \\
& =\phi^{A B} \epsilon^{\dot{A} \dot{B}}+\bar{\phi}^{\dot{A} \dot{B}} \epsilon^{A B} \in(1,0) \oplus(0,1),
\end{aligned}
$$

with left-handed part $\phi^{A B}=-F_{-}^{0 i} \sigma_{i}^{A B}$ and the right-handed part its complex conjugate.
[1] C. Rovelli and F. Vidotto, Covariant Loop Quantum Gravity: An Elementary Introduction to Quantum Gravity and Spinfoam Theory (Cambridge University Press, Cambridge, 2014).

[2] L. Freidel and S. Speziale, Twisted geometries: A geometric parametrisation of SU(2) phase space, Phys. Rev. D 82, 084040 (2010).

[3] C. Rovelli and S. Speziale, On the geometry of loop quantum gravity on a graph, Phys. Rev. D 82, 044018 (2010).

[4] L. Freidel and E. R. Livine, The fine structure of SU(2) intertwiners from $\mathrm{U}(\mathrm{N})$ representations, J. Math. Phys. (N.Y.) 51, 082502 (2010).

[5] E. Bianchi, P. Dona, and S. Speziale, Polyhedra in loop quantum gravity, Phys. Rev. D 83, 044035 (2011).

[6] M. Rocek and R. M. Williams, The quantization of Regge calculus, Z. Phys. C 21, 371 (1984).
[7] R. Penrose and M. A. H. MacCallum, Twistor theory: An Approach to the quantization of fields and space-time, Phys. Rep. 6, 241 (1973).

[8] J. Engle, E. Livine, R. Pereira, and C. Rovelli, LQG vertex with finite Immirzi parameter, Nucl. Phys. B799, 136 (2008).

[9] B. Dittrich and J. P. Ryan, Phase space descriptions for simplicial 4d geometries, Classical Quantum Gravity 28, 065006 (2011).

[10] F. Anzà and S. Speziale, A note on the secondary simplicity constraints in loop quantum gravity, Classical Quantum Gravity 32, 195015 (2015).

[11] K. P. Tod, Some symplectic forms arising in twistor theory, Rep. Math. Phys. 11, 339 (1977).

[12] A. Bette and S. Zakrzewski, Massive relativistic particles with spin and the two twistor phase space, in Proceedings of 
12th Workshop on Soft Physics-Hadrons 96, Crimea. Pub. Nat. Acad. of Sci. of Ukraine, Kiev, 1996 (2004), p. 336, arXiv:hep-th/0404024.

[13] S. Speziale and W. M. Wieland, The twistorial structure of loop-gravity transition amplitudes, Phys. Rev. D 86, 124023 (2012).

[14] W. M. Wieland, Twistorial phase space for complex Ashtekar variables, Classical Quantum Gravity 29, 045007 (2012).

[15] E. R. Livine, S. Speziale, and J. Tambornino, Twistor networks and covariant twisted geometries, Phys. Rev. D 85, 064002 (2012)

[16] E. R. Livine, Projected spin networks for Lorentz connection: Linking spin foams and loop gravity, Classical Quantum Gravity 19, 5525 (2002).

[17] M. Dupuis and E. R. Livine, Lifting SU(2) spin networks to projected spin networks, Phys. Rev. D 82, 064044 (2010).

[18] C. Rovelli and S. Speziale, Lorentz covariance of loop quantum gravity, Phys. Rev. D 83, 104029 (2011).

[19] J. Engle, R. Pereira, and C. Rovelli, The Loop-QuantumGravity Vertex-Amplitude, Phys. Rev. Lett. 99, 161301 (2007).

[20] L. Freidel and K. Krasnov, A new spin foam model for 4d gravity, Classical Quantum Gravity 25, 125018 (2008).

[21] E. R. Livine and S. Speziale, Consistently solving the simplicity constraints for spinfoam quantum gravity, Europhys. Lett. 81, 50004 (2008).

[22] M. Dupuis, L. Freidel, E. R. Livine, and S. Speziale, Holomorphic Lorentzian simplicity constraints, J. Math. Phys. (N.Y.) 53, 032502 (2012).

[23] W. M. Wieland, Complex Ashtekar variables and reality conditions for Holst's action, Ann. Inst. Henri Poincaré 13, 425 (2012).

[24] S. Speziale and M. Zhang, Null twisted geometries, Phys. Rev. D 89, 084070 (2014).

[25] L. Freidel and S. Speziale, From twistors to twisted geometries, Phys. Rev. D 82, 084041 (2010).

[26] B. Dittrich and S. Speziale, Area-angle variables for general relativity, New J. Phys. 10, 083006 (2008).

[27] S. Alexandrov, The new vertices and canonical quantization, Phys. Rev. D 82, 024024 (2010).

[28] C. Rovelli, Ashtekar formulation of general relativity and loop space nonperturbative quantum gravity: A Report, Classical Quantum Gravity 8, 1613 (1991).

[29] L. Freidel and J. Hnybida, A discrete and coherent basis of intertwiners, Classical Quantum Gravity 31, 015019 (2014).

[30] H. M. Haggard, Pentahedral volume, chaos, and quantum gravity, Phys. Rev. D 87, 044020 (2013).

[31] B. Dittrich and J. P. Ryan, Simplicity in simplicial phase space, Phys. Rev. D 82, 064026 (2010).

[32] H. M. Haggard, C. Rovelli, W. Wieland, and F. Vidotto, The spin connection of twisted geometry, Phys. Rev. D 87, 024038 (2013).

[33] J. W. Barrett, R. Dowdall, W. J. Fairbairn, F. Hellmann, and R. Pereira, Lorentzian spin foam amplitudes: Graphical calculus and asymptotics, Classical Quantum Gravity 27, 165009 (2010).

[34] F. Hellmann and W. Kaminski, Holonomy spin foam models: Asymptotic geometry of the partition function, J. High Energy Phys. 10 (2013) 165.
[35] V. Bonzom, Spin foam models for quantum gravity from lattice path integrals, Phys. Rev. D 80, 064028 (2009).

[36] S. Alexandrov, Simplicity and closure constraints in spin foam models of gravity, Phys. Rev. D 78, 044033 (2008).

[37] C. Rovelli, Simplicity and closure constraints in spin foam models of gravity, Discretizing parametrized systems: The magic of Ditt-invariance, arXiv:1107.2310.

[38] W. Kaminski, M. Kisielowski, and J. Lewandowski, Spinfoams for all loop quantum gravity, Classical Quantum Gravity 27, 095006 (2010).

[39] Y. Ding, M. Han, and C. Rovelli, Generalized spinfoams, Phys. Rev. D 83, 124020 (2011).

[40] W. M. Wieland, New action for simplicial gravity in four dimensions, Classical Quantum Gravity 32, 015016 (2015).

[41] E. F. Borja, L. Freidel, I. Garay, and E. R. Livine, U(N) tools for loop quantum gravity: The return of the spinor, Classical Quantum Gravity 28, 055005 (2011).

[42] M. Han and M. Zhang, Asymptotics of spinfoam amplitude on simplicial manifold: Lorentzian theory, Classical Quantum Gravity 30, 165012 (2013).

[43] L. Freidel and J. Ziprick, Spinning geometry $=$ Twisted geometry, Classical Quantum Gravity 31, 045007 (2014).

[44] M. Dupuis and E. R. Livine, Holomorphic simplicity constraints for $4 \mathrm{~d}$ spinfoam models, Classical Quantum Gravity 28, 215022 (2011).

[45] M. Dupuis and E. R. Livine, Holomorphic simplicity constraints for 4d Riemannian spinfoam models, J. Phys. Conf. Ser. 360, 012046 (2012).

[46] V. Bonzom, F. Costantino, and E. R. Livine, Duality between spin networks and the 2D Ising model, Commun. Math. Phys. 344, 531 (2016).

[47] A. Banburski, L. Q. Chen, L. Freidel, and J. Hnybida, Pachner moves in a 4d Riemannian holomorphic Spin Foam model, Phys. Rev. D 92, 124014 (2015).

[48] B. Dittrich and J. Hnybida, Ising Model from Intertwiners, arXiv:1312.5646.

[49] J. W. Barrett and L. Crane, A Lorentzian signature model for quantum general relativity, Classical Quantum Gravity 17, 3101 (2000).

[50] R. Sorkin, Time evolution problem in Regge calculus, Phys. Rev. D 12, 385 (1975).

[51] B. Bahr and B. Dittrich, Improved and perfect actions in discrete gravity, Phys. Rev. D 80, 124030 (2009).

[52] M. Han, Cosmological constant in LQG vertex amplitude, Phys. Rev. D 84, 064010 (2011).

[53] T. Thiemann, Quantum spin dynamics (QSD), Classical Quantum Gravity 15, 839 (1998).

[54] L. Freidel and J. Ziprick (unpublished).

[55] T. A. Koslowski, Loop quantization of shape dynamics, Proceedings of 13th Marcel Grossmann Meeting on Recent Developments in Theoretical and Experimental General Relativity, Astrophysics, and Relativistic Field Theories (MG13): Stockholm, Sweden, 2012 (2015), arXiv:1302.7037.

[56] V. Bonzom, M. Dupuis, F. Girelli, and E. R. Livine, Deformed phase space for 3d loop gravity and hyperbolic discrete geometries, arXiv:1402.2323.

[57] H. M. Haggard, M. Han, and A. Riello, Encoding Curved Tetrahedra in Face Holonomies: A Phase Space of Shapes 
from Group-Valued Moment Maps, Ann. Inst. Henri Poincaré 17, 2001 (2016).

[58] F. Luo, Combinatorial yamabe flow on surfaces, arXiv: math/0306167.

[59] D. Glickenstein, Discrete conformal variations and scalar curvature on piecewise flat two and three dimensional manifolds, J. Diff. Geom. 87, 201 (2011).

[60] A. Marzuoli and D. Merzi, Conformal variations and quantum fluctuations in discrete gravity, Int. J. Geom. Methods Mod. Phys. 13, 1650084 (2016).

[61] E. R. Livine, Deformation operators of spin networks and coarse-graining, Classical Quantum Gravity 31, 075004 (2014).

[62] S. Ariwahjoedi, J. S. Kosasih, C. Rovelli, and F. P. Zen, How many quanta are there in a quantum spacetime?, Classical Quantum Gravity 32, 165019 (2015).

[63] B. Bahr, B. Dittrich, and S. Steinhaus, Perfect discretization of reparametrization invariant path integrals, Phys. Rev. D 83, 105026 (2011).
[64] E. Bianchi, L. Hackl, and N. Yokomizo, Entanglement entropy of squeezed vacua on a lattice, Phys. Rev. D 92, 085045 (2015).

[65] A. Hamma, L.-Y. Hung, A. Marciano, and M. Zhang, Area law from loop quantum gravity, arXiv:1506.01623.

[66] A. Feller and E. R. Livine, Ising spin network states for loop quantum gravity: A toy model for phase transitions, Classical Quantum Gravity 33, 065005 (2016).

[67] A. Ghosh and D. Pranzetti, CFT/gravity correspondence on the isolated horizon, Nucl. Phys. B889, 1 (2014).

[68] L. Freidel and A. Perez, Quantum gravity at the corner, arXiv:1507.02573.

[69] V. Bonzom and B. Dittrich, 3D holography: From discretum to continuum, J. High Energy Phys. 03 (2016) 208.

[70] R. Penrose and W. Rindler, Spinor And Twistor Methods In Space-Time Geometry, Spinors and Space-Time (Cambridge University Press, Cambridge, England, 1986), Vol. 2. 